Mitteilungen der Österreichischen Geographischen Gesellschaft,

152. Jg. (Jahresband), Wien 2010, S. 130-162

\title{
Flachgründiger Abtrag auf Wiesen- und Weideflächen in Den Alpen (Blaiken) \\ - Wissensstand, Datenbasis und Forschungsbedarf
}

\author{
Christoph WIEgAND und Clemens GeITNER, beide Innsbruck*
}

mit 4 Abb. und 3 Tab. im Text

\section{INHALT}

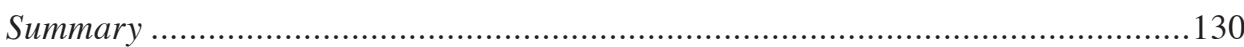

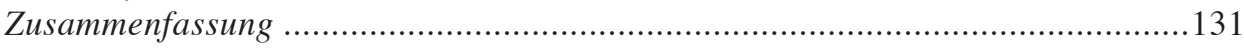

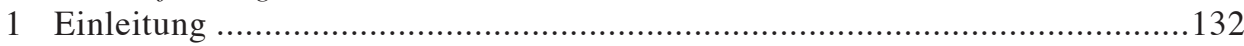

2 Teilprozesse der flachgründigen Denudation ........................................... 140

3 Steuernde Faktoren für flachgründige Massenbewegungen

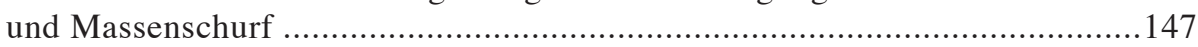

4 Synthese, offene Fragen und Forschungsbedarf ....................................... 156

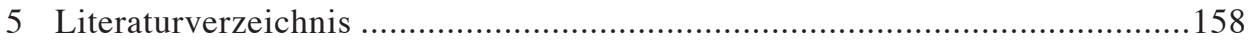

\section{Summary}

Shallow Erosion on Grasslands and Pastures of the Alps (Blaiken) - State of the Art, Data Base and Demand for Research

In recent decades, there has been an increased occurrence of shallow erosion on grasslands and pastures of the Alps, the so-called Blaiken. A synopsis of studies, which approached the phenomenon Blaike from diverse methodological angles - from extensive field and laboratory work to complex GIS-supported analysis - revealed agreement on several aspects, i.e. this type of erosion occurs as the displacement of a small area $\left(2-200 \mathrm{~m}^{2}\right)$ at a depth of two meters maximum. Even though each incidence represents a comparatively small dislocation of material, the total number of such erosion events amounts to a considerable loss of soil material. If the displacement occurs in form of translational landslides, it belongs to the process group

* Dipl.-Geogr. Christoph WIEgAnd, Institut für Gebirgsforschung: Mensch und Umwelt, Österreichische Akademie der Wissenschaften, Technikerstraße 21a, Otto-Hittmair-Platz 1, A-6020 Innsbruck; E-mail: christoph.wiegand@oeaw.ac.at,http://www.oeaw.ac.at/igf; Dr. Clemens GEITNER, Institut für Geographie, Leopold-Franzens-Universität Innsbruck, Innrain 52, A-6020 Innsbruck, Institut für Gebirgsforschung: Mensch und Umwelt, Österreichische Akademie der Wissenschaften; E-mail: clemens.geitner@uibk. ac.at, http://www.uibk.ac.at/geographie, http://www.oeaw.ac.at/igf 
of gravitational mass movements. Normally, the occurrence requires a preformed gliding horizon in the underground, which is often situated at the boundary between layers of different physical properties (different material, bulk density, etc.). Snow gliding can cause shallow erosion in the form of a superficial erosion process. Sward damages caused by trampling also belong to this type of shallow erosion. The most crucial parameters are topography (slope angle, aspect, elevation a.s.l.), climatic and geological settings, soil conditions, vegetation and land use. Individual studies differ considerably in their assessment of these factors, dependent on the characteristics of the study area and the research focus. In each case, it is the critical interaction of the several parameters that leads to erosion, which makes it particularly difficult to deduce relevant boundary values.

A study of the literature also reveals differing concepts of the processes involved. A clarification of the interdependences at work is hampered by a lack of reliable hydro(geo)logical, substrate and soil data. More detailed studies with a combination of geophysical measurements and high-resolution relief data could improve our understanding of these processes and their prognosis. A systematic survey of results from various regions of the Alps is needed to capture the critical interaction of factors and their spatial variability. Only a broad and comparable data base allows reliable conclusions on spatial and temporal aspects, which will become more significant for future decisions. In addition, we need to take a closer look at the effects of widespread land-use changes, as the increase in this type of erosion seems to be caused by the decrease in cultivation. Research is required on further developments in existing eroded areas as the total amounts of abrasion are considerable. Only systematic monitoring, as demanded by the Alpine Convention, can adequately capture this complex process and its potential changes, ideally complemented by interdisciplinary studies on various scales.

\section{Zusammenfassung}

Innerhalb der letzten Jahrzehnte lässt sich ein vermehrtes Auftreten flachgründiger Abtragungserscheinungen - sogenannter Blaiken - auf Wiesen- und Weideflächen der Alpen verzeichnen. Eine Zusammenschau der Studien, die sich mithilfe diverser methodischer Ansätze - von aufwändiger Gelände- und Laborarbeit bis zu komplexen GIS-gestützten Analysen - dem Phänomen Blaike widmeten, zeigt folgende Übereinstimmungen: Es handelt sich bei dieser Denudationsform um einen Abtrag geringer Flächengröße (2-200 $\left.\mathrm{m}^{2}\right)$ und einer Tiefe von maximal zwei Metern. Einzelereignisse führen lediglich zu einem geringen Materialversatz, in der Summe ergeben sich jedoch erhebliche Verluste von Bodenmaterial. Wenn der Abtrag in Form von Translationsrutschungen erfolgt, fällt er in den Prozessbereich gravitativer Massenbewegungen. Dafür bedarf es in der Regel einer präformierten Gleitschicht, die sich an Material-bzw. Lagerungsgrenzen im Untergrund orientiert. Es können aber auch Gleitschneeprozesse zur Ausbildung flachgründiger Denudation führen; diese gehören dann in den Prozessbereich des Massenschurfs. Trittverletzungen der Grasnarbe durch Weidevieh werden ebenfalls den Blaiken zugeschlagen. 
Als steuernde Parameter sind die Topographie (Hangneigung, Exposition und Höhe), die klimatischen und geologischen Bedingungen (Lockersubstrataufbau), der Boden, die Vegetation und die Nutzung eines Standortes entscheidend. In den einzelnen Studien werden diese Faktoren mitunter sehr verschieden gewichtet, was nicht nur durch die unterschiedlichen Lokalitäten, sondern auch durch den fachspezifischen Fokus bedingt scheint. Weil im Einzelfall immer eine spezielle, kritische Überlagerung mehrerer Parameter zum Abtrag führt, ist die Ableitung relevanter Grenzwerte kaum möglich.

Auch in Bezug auf das Prozessverständnis zeigen sich in der Literatur abweichende Auffassungen. Für eine umfassendere Klärung der Zusammenhänge fehlt es oftmals an verlässlichen hydro(geo)logischen sowie substrat- und bodenphysikalischen Daten. Weitere Detailstudien, in denen geophysikalische Messungen und hoch aufgelöste Reliefdaten kombiniert werden, könnten das Prozessverständnis und damit auch die Prognostizierbarkeit weiter verbessern. Um die kritischen Faktorenkombinationen und ihre räumliche Variabilität besser beschreiben zu können, sollte eine systematische Zusammenstellung von Einzelereignissen aus weiten Teilen des Alpenraums angestrebt werden. Nur eine breite und vergleichbare Datenbasis ermöglicht verlässliche räumliche und zeitliche Aussagen, die für zukünftige Entscheidungen immer wichtiger werden.Zudem müssen die Effekte des weit verbreiteten Nutzungswandels noch genauer untersucht werden, denn die Zunahme dieser Denudationsform ist offensichtlich auch durch den Rückgang der Bewirtschaftung bedingt. Forschungsbedarf besteht ebenso im Hinblick auf die Weiterformung existierender Erosionsflächen, da die hierbei erreichten Abtragssummen nicht unerheblich sind. Um dem komplexen Prozessgefüge und seinen möglichen Änderungen gerecht werden zu können, bedarf es schließlich-neben weiteren interdisziplinären Studien auf unterschiedlichen Maßstabsebenen - eines systematischen Monitorings, wie es auch die Alpenkonvention nachdrücklich fordert.

\section{Einleitung}

\subsection{Relevanz und Aktualität des Themas}

Flachgründiger Abtrag stellt einen Prozess in den Alpen dar, dem, verglichen mit großen Massenbewegungen wie Fels- und Bergstürzen, kaum öffentliche Aufmerksamkeit zuteil wird, kommt es doch zu keinen gravierenden Zerstörungen von Infrastruktur oder gar zu Verlusten von Menschenleben. Aber auch wenn die einzelne Fläche nur einen relativ geringen Materialabtrag aufweist, ist durch das gehäufte Auftreten dieser Prozesse in der Summe ein erheblicher Verlust von Boden zu verzeichnen. Für Tirol hat GALL diesen Tatbestand in seiner Dissertation bereits 1947 dargelegt und räumlich differenziert (GALl 1947). Seit rund zehn Jahren hat der Schutz der Ressource Boden durch das Protokoll „Bodenschutz“ Eingang in die Alpenkonvention gefunden und durch die Ratifizierung im gesamten Alpenraum Rechtsverbindlichkeit erlangt (CIPRA 1998). Die damit verbundenen Forderungen reichen von der Intensivierung und internationalen Harmonisierung der Bodenforschung über den Ausbau der bodenrelevanten Datenbasis bis zum Aufbau eines Bodenmonitorings (Geitner \& Tusch 2008). Der 
vorliegende Beitrag soll anhand eines zentralen Aspektes der Bodengefährdung diesen noch weitgehend unerfüllten Forderungen nachkommen, indem er den Wissensstand und die Datenbasis umreißt und den daraus abzuleitenden Forschungsbedarf ausweist.

In den vergangenen Jahren wurde eine Reihe von Untersuchungen zum flachgründigen Materialversatz auf Wiesen- und Weideflächen in der montanen bis alpinen Stufe des Alpenraums unternommen. Dabei wurden unterschiedliche Nutzungsintensitäten sowie diverse Sukzessionsstadien auf Brachflächen berücksichtigt. Eine Motivation für diese Studien dürfte auch darin gelegen haben, dass sich das Auftreten dieser Denudationsformen während der letzten Dekaden vermehrt zu haben scheint. Eine Auswahl von Arbeiten zu diesem Thema und ihre räumliche Zuordnung sind in Tabelle 1 zusammengestellt und in Abbildung 1 lokalisiert. Dabei handelt es sich ausschließlich um Fallstudien in unterschiedlichen und zudem verschieden großen Untersuchungsgebieten. Überblicksarbeiten, die wie bei BiRKEnHAUER (2001) auch regionale Vergleiche beinhalten, finden sich dagegen kaum.

\begin{tabular}{|c|c|c|c|}
\hline Naturraum & Region & Autor & Untersuchungsgebiet \\
\hline \multirow[t]{3}{*}{ Ostalpen } & Nördl. Teil & $\begin{array}{l}\text { ANDRECS et al. 2002, } \\
\text { MARKART et al. } 2007 \\
\text { BLECHSCHMIDT } 1990 \\
\text { BUNZA et al. } 1996 \\
\text { EIDT \& LÖHMANNSRÖBEN } 1996 \\
\text { KARL } 1961 \\
\text { KELCH et al. } 1977 \\
\text { LAATSCH \& GROTTENTHALER } \\
1973 \\
\text { MÖSSMER 1984, } 1985 \\
\text { RIEDL } 1982 \\
\text { SCHAUER } 1975 \\
\text { STAHR } 1997 \\
\text { ZWECKL \& SPANDAU } 1987\end{array}$ & $\begin{array}{l}\text { Verschiedene UG* in Vorarlberg } \\
\text { Karwendel } \\
\text { Nordalpen }\left(23 \mathrm{WEZG}^{* *}\right) \\
\text { Nordalpen }(23 \text { WEZG**) } \\
\text { Allgäuer Alpen } \\
\text { Chiemgauer Berge } \\
\text { Landkreis Miesbach } \\
\text { Landkreis Miesbach \& Rosenheim } \\
\text { Sameralm, Tennengebirge } \\
\text { Berchtesgadener, Allgäuer } \\
\text { Alpen } \\
\text { Nationalpark Berchtesgaden } \\
\text { Nationalpark Berchtesgaden }\end{array}$ \\
\hline & Zentr. Teil & $\begin{array}{l}\text { BERNHAUPT } 1980 \\
\text { STOCKER } 1985,2005 \\
\text { TASSER \& TAPPEINER } 2002 \\
\text { TASSER et al. } 1999,2005 \mathrm{a}\end{array}$ & $\begin{array}{l}\text { Planneralm, Wölzer Tauern } \\
\text { Kreuzeckgruppe, Kärnten } \\
\text { Stubaital \& Passeiertal }\end{array}$ \\
\hline & Südl. Teil & $\begin{array}{l}\text { CERIANI et al. } 1992 \\
\text { TASSER et al. 2003, 2005a,b }\end{array}$ & $\begin{array}{l}\text { Lombardei [Lombardia] } \\
\text { Monte Bondone, Trient [Trento] }\end{array}$ \\
\hline Westalpen & $\begin{array}{l}\text { Zentr. Teil } \\
\text { Südl. Teil }\end{array}$ & $\begin{array}{l}\text { Rickli \& Graf } 2009 \\
\text { Meusburger \& Alewell } \\
2008\end{array}$ & $\begin{array}{l}6 \mathrm{UG}^{*} \text { in der Schweiz } \\
\text { Urseren Tal, Schweiz }\end{array}$ \\
\hline
\end{tabular}

* UG = Untersuchungsgebiete, $* *$ WEZG $=$ Wildbacheinzugsgebiete

Tab. 1: Ausgewählte Arbeiten zu flachgründigem Abtrag auf Wiesen- und Weideflächen in den Alpen mit räumlicher Zuordnung 


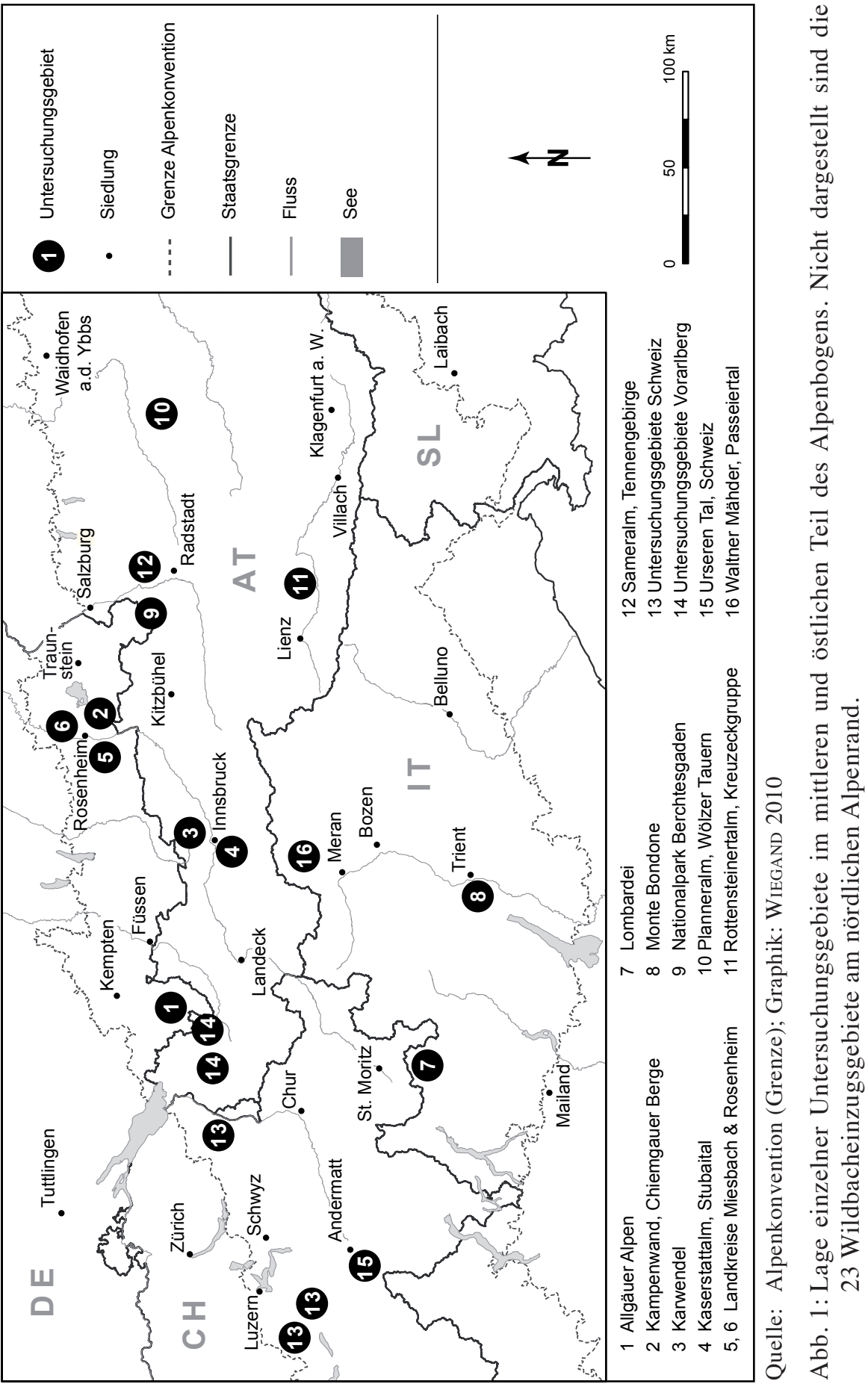


Neben den Untersuchungen im Alpenraum gibt es zahlreiche Arbeiten aus anderen Gebirgsräumen, die vergleichbare Prozesse behandeln (z.B. Apuanische Alpen: GianneCChini 2005; Pyrenäen: Begueria 2006, Garcia-Ruiz et al. 1996; Japan: Hiura 1988, Marui 1984; Neuseeland: Brooks et al. 2002, deRose et al. 1995, Douglas et al. 1986, GLADE 1998).

Wissenschaftlicher Bedarf bei der Untersuchung flachgründiger Abtragung besteht vor allem noch im Hinblick auf das Prozessverständnis und - was damit im Zusammenhang steht - die Klärung der Begrifflichkeiten. Zudem wäre auf Grundlage einer möglichst vollständigen Zusammenstellung und Auswertung der bisherigen Arbeiten eine vorläufige Synthese anzustreben, bei der auch die bestehenden Widersprüche und offenen Fragen herausgearbeitet werden. Dabei sollte auch der großen naturräumlichen Variabilität der vorliegenden Studien Rechnung getragen werden. Mit dem vorliegenden Beitrag soll ein erster Versuch in diese Richtung unternommen werden.

Der globale Wandel - seien es die Veränderungen des Klimas oder der Landnutzung - fällt in Gebirgen in der Regel deutlicher aus als in anderen Landschaften der Erde (BECKER et al. 2007, SALA et al. 2000; UNCED 1992, S. 124). Das lässt sich auch für die Alpen feststellen (PArry et al. 2007, S. 552; Psenner 2008, S. 26; Veit 2002, S. 276), wobei der sowohl tiefgreifende als auch weit verbreitete Nutzungswandel zurzeit die dominante Rolle spielt (MACDonald et al. 2000). Dieser hat auch einen nachweislichen Einfluss auf Wasserhaushalt und Morphodynamik (TASSER et al. 2003, 2005a, b), sodass es methodisch kaum möglich ist, die morphodynamischen Auswirkungen anthropogener Einflüsse von womöglich klimainduzierten Effekten zu trennen oder gar ihre Anteile annähernd zu quantifizieren. Gerade vor diesem Hintergrund erscheint es relevant, das geomorphologische Prozessverständnis weiter zu vertiefen, denn nur auf dieser Grundlage können verlässlichere Prognosen abgegeben und mögliche Empfehlungen für die zukünftige Landnutzung ausgesprochen werden. Zudem besteht dringender Bedarf, ein langfristig angelegtes Monitoring dieser Denudationsprozesse aufzubauen. Auch hierzu bedarf es genauerer Prozesskenntnis, um gezielt nur die Daten erheben zu können, die für vergleichende Auswertungen - sowohl räumlich als auch multitemporal - relevant sind.

\subsection{Begriffsdefinitionen und -abgrenzungen}

Unterschiedliche Formen des flachgründigen Abtrags werden in weiten Teilen des deutschsprachigen Alpenraums als Blaiken bezeichnet. Da diese eher umgangssprachliche Bezeichnung bereits um die vorletzte Jahrhundertwende auch in die wissenschaftliche Literatur Eingang fand (PENCK 1894, GöTZINGER 1907 sowie STINy 1910, zitiert nach STOCKER 1985, S. 44), soll sie an erster Stelle erläutert werden. Etymologisch weist der Begriff Blaike [auch Plaike: von blaicken, blecken = blankes oder weißes entblößen] auf vegetationslose bis schütter bewachsene Abtragungsformen oder Schädigungen der Bodendecke an Berghängen hin (STAHR 1997, S. 8). Diese Abtragsformen erreichen nach Bernhaupt (1980, S. 298) und Schauer (1975, S. 1) zumeist nur Ausdehnungen von 2 bis $200 \mathrm{~m}^{2}$, wobei es nicht sinnvoll erscheint, großflächigere Formen prinzipiell auszuklammern. Ihre Tiefe wird in der Regel nur mit wenigen Dezimetern, maximal 

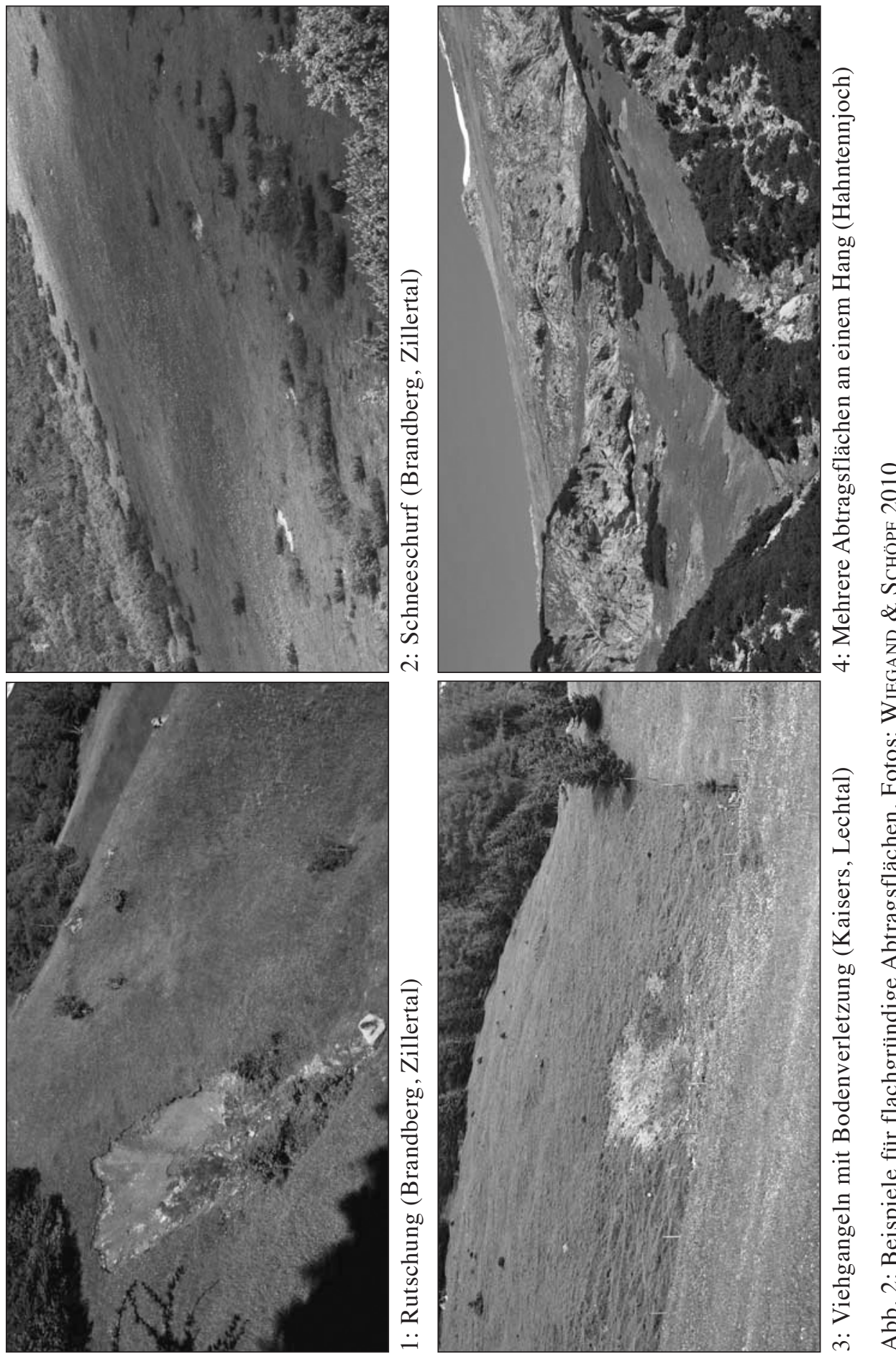

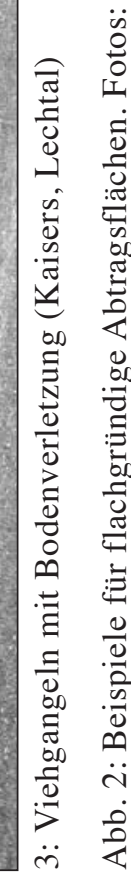


aber zwei Metern angegeben. Damit ist diese Prozessgruppe auf den oberflächennahen Bereich begrenzt. Abbildung 2 zeigt Blaiken im Sinne dieser Definition, die jedoch unterschiedlich entstanden sind (vgl. Kap. 2).

Dem morphologisch recht eindeutig charakterisierbarem Phänomen der Blaike werden unterschiedliche Prozesse zugewiesen: Nach den Untersuchungen zum Beispiel von Schauer (1975) handelt es sich um einen gravitativen Prozess, der durch die Einwirkung des Schneeschurfs lediglich vorbereitet wird. STAHR (1997) hingegen sieht im Auftreten von Blaiken eine Form des Massenschurfs, als unmittelbare Folge abgehender Schneebretter und Lawinen. Während die beiden erstgenannten - wie auch die meisten anderen - Autoren die Ausbildung von Blaiken innerhalb des Lockermaterialkörpers beschreiben, untersucht STOCKER $(1985,2005)$ auch die Bildung von Blaiken bis in das anstehende Gestein, welche dann im Wesentlichen durch Frostverwitterung bestimmt ist. Im Hinblick auf die Auslösung der Prozesse beschreiben MAAG et al. (2001, S. 25) Blaikentypen, die durch Viehtritt hervorgerufen werden. RIEDL (1982) zeigt auf, dass auch anthropogen-technische Eingriffe - wie der Wegebau - die Blaikenbildung initialisieren können. Der unterschiedlichen Genese entsprechend liegt auch eine Vielfalt an Blaiken-Bezeichnungen vor, wie beispielsweise Schneeschurfblaike, Narbenversatz, Narbenversatzblaike, Trittblaike und Wegblaike (BERNHAUPT 1980, S. 291; LAATSCH \& Grottenthaler 1972, S. 331; Riedl 1982, S. 23ff.).

Für diese Art von Formen und Prozessen findet sich in der Literatur auch eine Reihe anderer Begriffe. So bezeichnet STAHR (1997, S. 9) beispielsweise den Blattanbruch als einen schichtweisen bzw. blattförmigen Bodenabtrag mit auffallend scharfer Abtragungsfront, des sogenannten Boden- oder Rasenkliffs mit Hohlkehlbildung. Die bei diesem Prozess aus dem Verband gelösten Boden- oder Rasenschollen verlagern sich hangabwärts. Unter Translations- oder Blatterdrutsch versteht man nach LAATSCH \& Grottenthaler (1972, S. 317) ebenfalls eine Verlagerung von Bodenschollen entlang einer wenige Dezimeter unterhalb der Erdoberfläche liegenden Schwächezone (vgl. Abb. 3).

Sowohl die flachgründigen Rutschungen als auch der Massenschurf sind der Denudation zuzuordnen, also den geomorphologischen Prozessen, die eher flächenhaft ablaufen und somit dem Begriff der Erosion, der sich auf linienhaft wirkende Prozesse bezieht, gegenüberstehen (AHNERT 1999, S. 120, 192; Zepp 2004, S. 77, 100). Fraglich ist hingegen, ob man diese Formen des Abtrags zum Prozess der Bodenerosion zählen sollte. Unter diesem versteht man nach ZEPP (2004, S. 131) eine durch den Menschen bedingte, übermäßige und oberflächige Abtragung durch Wasser und Wind bzw. nach AHNERT (1999, S. 398) die vom Menschen verursachte oder beschleunigte Abtragung von Regolithmaterial (Regolith = Verwitterungsdecke) insbesondere durch Flächen- und Rillenspülung. In diesem Sinne zählten die hier behandelten Prozesse nicht zur Bodenerosion, obwohl sie zu erheblichen Bodenverlusten führen können. AuERSwALD (1998, S. 61) berücksichtigt zwar diese Formen des Bodenabtrags durch schwerkraftbedingte Prozesse, bezeichnet sie aber gesondert als gravitative Bodenverlagerung. Auch Morgan (2005, S. 37) weist darauf hin, dass neben äolischen und fluvialen Prozessen ebenso Massenbewegungen zu Bodenverlusten führen und betont, dass diese oft vernachlässigt werden. Etwas weiter geht Auerswald in Scheffer \& Schachtschabel (2002, S. 416), indem er gravitative Prozesse sowie Bodenverluste 


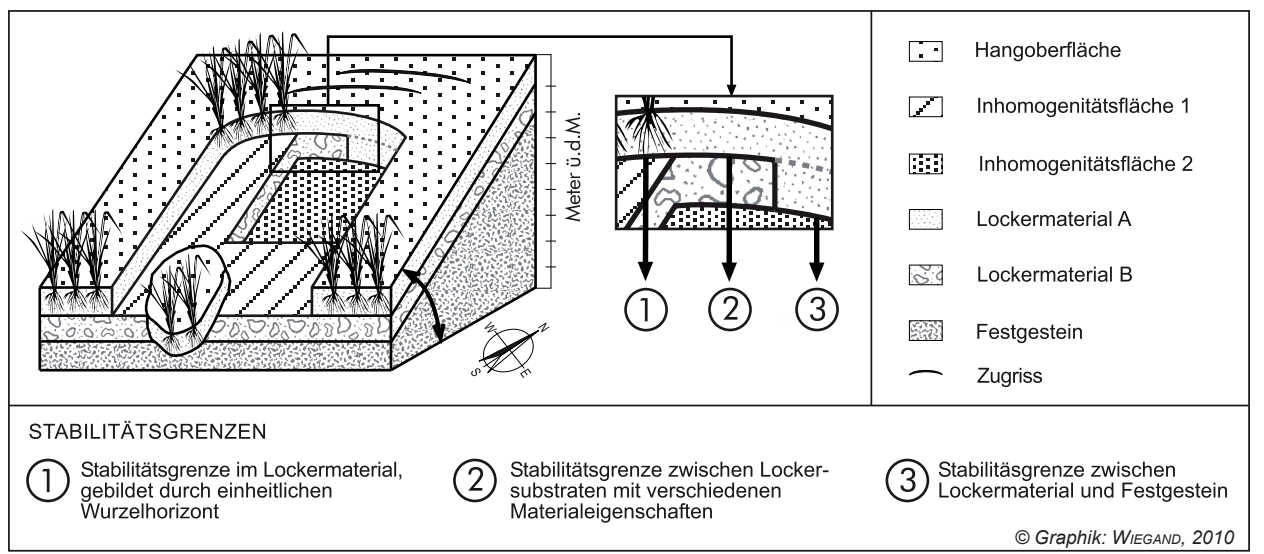

Abb. 3: Schematische Darstellungen einer rutschungsbedingten Blaike

Dargestellt ist der Versatz einer Materialscholle auf einer Inhomogenitätsschicht entlang eines einheitlichen Wurzelhorizontes (1) und entlang einer Stabilitätsgrenze im Lockermaterial (2). Nicht dargestellt ist das Abgleiten einer Scholle über dem anstehenden Gestein. Des Weiteren sind drei maßgebliche Reliefparamenter skizziert: Hangneigung, Höhe und Exposition.

durch Schneeschurf eindeutig zur Bodenerosion hinzu zählt. Aus Sicht der Autoren erscheint es durchaus angebracht, die flachgründige Abtragung durch Massenbewegungen oder Massenschurf in den Begriff der Bodenerosion mit einzubinden, denn diese sind nicht nur mit erheblichen Bodenverlusten verbunden, sondern treten vor allem auf Flächen landwirtschaftlicher (Grünland-)Nutzung auf.

In der englischen Fachsprache werden diese Prozesse dennoch dem Begriff erosion zugeordnet, da dieser weiter gefasst ist und in der Regel Denudationsvorgänge mit einschließt (AHNert 1999, S. 192). Die gravitativen Typen der in Frage stehenden Abtragsformen und -prozesse werden meist mit dem Terminus shallow landslides bezeichnet. Dieser beschränkt sich nach BRooks et al. (2002, S. 166), Dymond et al. (1999, S. 122) und LAteltin et al. (2005, S. 313) auf flachgründige Rutschungen an Hängen bis in maximal zwei Meter, nach CAINE (1980, S. 23) bis in drei Meter Tiefe. Damit ist klar, dass sich die Rutschung sowohl im oberflächennahen Boden als auch im mächtigeren Lockergestein ausbilden kann. Es finden sich weitere, ähnlich gebrauchte Bezeichnungen wie slab slide, sheet slide oder flake slide (DIKAU 2004, S. 647; IBSEN \& BRUnSDEN 1996, S. 78). Wenn sich die Massenverlagerung hingegen auf den eigentlichen Boden beschränkt, wird der Ausdruck soil slip oder shallow translational failure in dry cohesionless soil verwendet (Douglas et al. 1986; GianneCCHini 2005, S. 21; Ibsen \& BRunsden 1996, S. 78). Für den Abtrag durch Schnee oder Viehtritt finden sich im Englischen zumeist nur Umschreibungen der auslösenden Prozesse (snow gliding processes bzw. livestock trampling).

Wie im Kapitel 2 noch zu zeigen sein wird, lassen sich die am Formenkomplex Blaike beteiligten Prozesse in die Gruppe der Massenbewegungen einerseits und die des Massenschurfs andererseits unterteilen. Dementsprechend differenziert müssen die Prozesse und möglichst auch die Formen betrachtet werden. 


\subsection{Verwendete Untersuchungsmethoden}

Zur Untersuchung der bei flachgründiger Denudation beteiligten Prozesse werden verschiedene Methoden verwendet. Zunächst können die Abtragsflächen kartiert sowie die topographischen, geologischen, geomorphologischen, bodenkundlichen und vegetationskundlichen Parameter der erodierten Fläche und ihrer unmittelbaren Umgebung aufgenommen werden. Zusätzliche Informationen zur aktuellen Landnutzung und möglichst auch zu historischen Landnutzungen sollten ebenfalls in die Erhebungen einfließen (BunZA et al. 1996). Zur experimentellen Untersuchung können unter anderem Beregnungsversuche (Bunza et al. 1996, MARKART et al. 2006), Schneegleitversuche (Leitinger et al. 2008, Newesely et al. 2000), Schneedruckmessungen (Blechschmidt 1990) sowie Abtragsmessungen mit Sedimentfallen (STOCкER 2005) durchgeführt werden. Den Aufnahmen im Feld können sich weiterführende Laboranalysen anschließen, die der Bestimmung bodenphysikalischer (Bodenstruktur, Textur, Dichte etc.) und bodenchemischer ( $\mathrm{pH}$-Wert, Humusgehalt, C/N-Verhältnis etc.) Parameter dienen. Um Aussagen über die zeitlichen Veränderungen der Denudationsstellen innerhalb eines Gebietes zu erhalten, bieten sich multitemporale Auswertungen von Luftbildern an (Alewell et al. 2008, Kelch et al. 1977). Mit Fernerkundung lassen sich generell Daten auch aus schwer zugänglichen Gebieten erheben. Zur Bestimmung von Massenverlagerungen werden auch terrestrische Laserscansysteme benutzt (ABELLAN et al. 2009, Bitelli et al. 2004). Vergangene Denudationsprozesse können mithilfe von Isotopenmessungen im Oberboden nachgewiesen und räumlich differenziert werden. Dem verwendeten Isotop entsprechend, lassen sich unterschiedliche zeitliche Dimensionen erschließen, mit $\mathrm{C}^{137}$ beispielsweise sind Ereignisse ab 1986 detektierbar (Alewell et al. 2008, Konz et al. 2009). Auch groß angelegte Feldexperimente, in denen künstlich ausgelöste Rutschungen detailliert untersucht werden, kommen zum Einsatz (Projekt CCES-TRAMM der Eidgenössischen Forschungsanstalt für Wald, Schnee und Landschaft).

Durch geostatistische Auswertungen der erhobenen Parameter kann deren Einfluss auf die Prozesse abgeschätzt werden. Dabei werden beispielsweise multivariate Berechnungen wie Regressionsanalysen verwendet (GAMPER \& TASSER 2002, HAMBERGER \& Moser 2008, Mössmer 1985). Die GIS-Technologie ermöglicht es, die verschiedenen Datensätze miteinander zu verschneiden, auszuwerten und darzustellen. Die Abtragsmodellierung (B ATHURST et al. 2010) hilft Prognosen zu erstellen, wie sich der gesellschaftliche und der klimatische Wandel weiterhin auswirken können (TAPPEINER et al. 1998, 2008).

Was neben diesen zahlreichen Forschungsansätzen noch weitgehend fehlt, sind Konzepte und deren Umsetzung für ein Monitoring dieser Prozesse. Auf lange Sicht aber würde ein gut angelegtes Monitoring die solideste Datengrundlage liefern, um einen möglichen Wandel der geomorphologischen Dynamik nachweisen zu können. 


\section{Teilprozesse der flachgründigen Denudation}

Um die Diskussion über die beteiligten Prozesse besser strukturieren zu können, werden diese nach Initialisierung, dem eigentlichen Materialtransport und der sekundären Weiterformung unterschieden.

\subsection{Initialisierung}

Häufig wird das Schneegleiten, also die Bewegung der Schneedecke auf dem Untergrund, als Initialisierung für den flachgründigen Abtrag betrachtet, da es hierbei zum Aufreißen der Bodendecke kommen kann. Begünstigt wird die Gleitschneebewegung durch das Aufkommen von langhalmigen Gräsern (z.B. Deschampsia caespitosa [L.] Beauv., Molinia arundinaceae Schrank., Carex sempervirens Vill.) - örtlich auch als Lahnergräser bezeichnet -, die sich unter der Last des Schnees umbiegen und ihm so eine glatte Rutschfläche bieten (Ewald 1996, S. 131; LAatsch \& Grottenthaler 1972, S. 329). Das Aufreißen des Untergrunds durch Gleitschnee wird allerdings laut SCHAUER (1975, S. 15) erst durch verstärktes Aufkommen horstbildender Gräser nach einer Flächenbrachlegung gefördert (z.B. Dactylis glomerata L., Nardus stricta L., Deschampsia cespitosa L.), da sich die Bewegung des Schnees durch die eingefrorenen Pflanzenteile auf den Untergrund übertrage. LAATSCH \& GrotTEnTHALER (1972, S. 330) beschreiben den Einfluss des Gleitschnees auf den oberflächennahen Untergrund ähnlich, betonen jedoch, dass der Widerstand der Horste in der Schneedecke genüge, um die Bewegung auf den Boden zu übertragen. Das Einfrieren von Pflanzenteilen wird von ihnen hingegen als unwahrscheinlich angesehen, da es hierfür zu einer Eis- bzw. Harschbildung in den bodennahen Schneeschichten kommen müsste. Diese würde aber dem Prozess des Schneegleitens entgegenwirken, denn dieser bedarf neben einer glatten Oberfläche und ausreichender Neigung auch einer bodennahen Nassschneeschicht (LaAtsch \& Grottenthaler 1972, S. 329) und Temperaturen über $0{ }^{\circ} \mathrm{C}$ (Newesely et al. 2000, S. 64). Es fehlt eine Diskussion darüber, inwieweit ein vermehrtes Auftreten sowohl von Pflanzenhorsten als auch von Zwergsträuchern durch einen erhöhten Reibungswiderstand das Schneegleiten einschränkt. Als dem Gleitprozess und der Bildung von Zugrissen förderlichste Disposition kann die Kombination von langhalmigen Gräsern und vereinzelten Pflanzenhorsten oder Zwergsträuchern angenommen werden. STAHR (1997, S. 114) kommt generell zu einer anderen Einschätzung, denn er konnte keinen Zusammenhang zwischen einer auffälligen Dominanz horstbildender Gräser und dem Aufkommen von Zugrissen und Blaiken feststellen.

Die nach der Ausaperung im Frühjahr beobachtbaren Risse in der Grasnarbe und dem Boden können eine Längserstreckung von mehreren Metern erreichen, sind im Durchschnitt aber nur wenige Zentimeter breit und reichen wenige Dezimeter tief in den Untergrund (Bernhaupt 1980, S. 301; SCHAuER 1975, S. 15). Sie ermöglichen das vermehrte Eindringen von Oberflächenwasser, welches so zum Entstehen von flachgründigen Rutschungen beitragen kann. Oft bilden sich oberhalb bestehender Denudationsflächen aufgrund der Destabilisierung erneut Zugrisse, und der Abtrag kann etappenweise hangaufwärts fortschreiten, was zur Erweiterung bereits geschädigter Flächen führt (AuERSWALD 1998, S. 63; RIEDL 1982). 
Als weiterer Prozess, der flachgründigen Abtrag auslösen kann, wäre der Viehtritt zu nennen. Auch in diesem Punkt muss genauer differenziert werden: Die Hufe schwerer Rinderrassen können Schädigungen der Grasnarbe hervorrufen, die zum Ansatzpunkt auch großflächiger Materialverlagerung werden können (LAATSCH \& GROTTENTHALER 1973, S. 19). Andererseits führt ein geordneter Weidegang mit begrenzter Bestoßungszahl und nicht zu schwerem Vieh dazu, dass bereits entstandene Zugrisse wieder zugetreten werden, was der Denudation entgegengewirkt (BERNHAUPT 1980; TASSER et al. 2005b). Ein weiterer erosionsmindernder Effekt ergibt sich aus der Terrassierung der Hänge durch ,isohypsenparallele zoogene Mikroformen linearer Prägung“ (RIEDL 1982, S. 9), den sogenannten Viehgangeln. Durch sie erhöht sich die Bodenrauhigkeit, wodurch dem Schnee ein größerer Reibungswiderstand entgegengebracht wird (BLECHSCHMIDT 1990; Schauer 1975, S. 18; TASSER et al. 2005b).

Neben den genannten Auswirkungen des Viehtritts auf den Boden beschreibt KARL (1961, S. 57) ein vermehrtes, streifenförmiges Auftreten von Deschampsia caespito$s a$ L. an den Viehgangeln von Schafen. Auch diese Bestände übertragen die Kräfte des Schneegleitens verstärkt auf den Boden. Es entstehen zunächst Zugrisse oberhalb der Kleinterrassen, die sich durch weitere Gleitbewegungen, Wasser und Frost erweitern, sodass die Terrassen in Folge herausgehoben und überkippt werden.

\subsection{Primärer Materialversatz}

Der eigentliche Materialversatz - zumeist als Abrutschen von Schollen - kann durch zwei Typen von Massenverlagerungen geschehen: Tritt der Transport ohne die Beteiligung eines Transportmittels aufgrund der exponierten Lage am Hang und der somit dominanten Schwerkraftkomponente ein, handelt es sich um eine gravitative Massenselbstbewegung (LaAtsch \& Grottenthaler 1972, S. 310). Zur Ausbildung eines solchen Ereignisses bedarf es in der Regel eines auslösenden Moments (trigger), z.B. in Form von Starkniederschlägen oder eines gesteigerten Oberflächenabflusses in Folge der Schneeschmelze. Ist die Materialverlagerung hingegen durch ein sich in Bewegung befindliches Medium bedingt, gehört der Prozess in die Gruppe des Massenschurfs respektive des Massentransports (LAATSCH \& GROTTENTHALER 1972, S. 329).

Im Fall der Massenselbstbewegung erfolgt die Ausbildung der Denudationsstellen gravitativ, d.h. Bodenschollen rutschen auf sich im Untergrund befindlichen, präformierten Inhomogenitäts- respektive Gleitflächen hangabwärts (vgl. Abb. 3). Die Auslöser, vor allem in Form von lang anhaltenden, ergiebigen Niederschlägen oder der Schneeschmelze, bewirken eine Wassersättigung des Lockermaterials, einhergehend mit einer Gewichtszunahme des Bodens und des Lockersubstrats und dem Aufbau eines positiven Porenwasserdrucks. Zudem kann das in vorhandene Zugrisse eingedrungene Wasser entlang von Inhomogenitätsflächen im Lockermaterial abfließen, wodurch die Reibung zwischen den Schichten reduziert wird und potenzielle Gleitflächen entstehen (Schauer 1975). Werden bestimmte, von den Materialeigenschaften abhängige Schwellenwerte überschritten, kommt es zum Abrutschen der oberen Schicht.

Die zur Rutschungsbildung beitragenden Inhomogenitätsflächen können sich entlang verschiedener Material- oder Lagerungsgrenzen bilden, z.B. an der Grenze vom 
Lockergestein zum darunter liegenden Festgestein. Auch innerhalb des Lockersubstrates finden sich geologisch-geomorphologisch bedingte Grenzen, beispielsweise bei den an Hängen weit verbreiteten, periglazialen Schuttdecken (STAHR 1997, S. 27; VeIT et al. 2002). Bestimmend ist hier vor allem ein Wechsel des Skelettgehalts und der Korngrößenverteilung der Feinerde, die sich über die innere Reibung und den Wasserhaushalt des Substrats auf dessen Stabilität auswirken. Die Bodenbildung orientiert sich am Aufbau dieser Schuttdecken, sodass Schichtgrenzen und Horizontgrenzen häufig übereinstimmen (VEIT 2002, S. 132).

Auch reine Horizontgrenzen können zu potenziellen Gleitflächen werden, wenn sich beispielsweise ein wasserdurchlässiger Horizont über einem wasserstauenden Horizont befindet (Avanzi et al. 2004). Nicht zuletzt können biogene Einflüsse zu Inhomogenitäten im Vertikalaufbau des Bodens führen, zum Beispiel durch die Bildung eines einheitlichen Wurzelhorizontes. Wenn alle Wurzeln in derselben Bodentiefe enden, geht unterhalb dieses Niveaus die Stabilität abrupt zurück (vgl. Abb. 3). Solche Beobachtungen konnten von SchaUER (1975) insbesondere bei aufgelassenen Flächen im nördlichen Alpenraum gemacht werden.

Im Gegensatz zu Schauer (1975) beschreibt StAHR (1997) die Bewegung des Schnees entlang der Bodenoberfläche nicht nur als vorbereitenden Prozess der Blaikenbildung, sondern er sieht darin den eigentlichen Treiber für den Abgang einer Bodenscholle. Denn, so die Argumentation, die sich plötzlich in Bewegung setzenden Schneebretter und Lawinen bewirken im durchnässten, frostfreien Boden - eine Situation, die häufig während der Schneeschmelze im Frühjahr auftritt - eine ebenso plötzliche Abnahme der Bodenstabilität, da sich durch die kurzzeitig stark erhöhte Auflast des Schnees wesentliche bodenphysikalische Zustände ändern. Das Porenvolumen im Boden wird aufgrund der Kompression verkleinert, sodass die stabilisierend wirkenden Oberflächenspannungen der Wassermenisken schwinden. Zusätzlich kann der Porenwasserdruck stark erhöht werden, was den Kontakt der Bodenteilchen mindert und somit den inneren Reibungswinkel herabsetzt (STAHR 1997, S. 109). Dass - obgleich der Prozess auf den gesamten Boden wirkt - letztlich Bodenschollen versetzt werden, wird durch die zusammenhaltende Wirkung der Wurzeln oberhalb der Gleitbahn hervorgerufen. Da die Schneedecke als Medium für die Materialverlagerung verantwortlich ist, muss der Prozess nach STAHR (1997) dem Massenschurf respektive -transport zugeordnet werden. Der beschriebene Effekt tritt vermehrt in sogenannten Gleitschneewintern auf. Durch die höheren Temperaturen an der Grenze der Schneedecke zum Untergrund bildet sich ein Gleitfilm durch flüssiges Wasser. Zudem ist in solchen Jahren nicht nur der Schnee stärker wassergesättigt und damit schwerer, sondern auch der Untergrund in der Regel feuchter. Neben dem Abgleiten ganzer Bodenschollen aufgrund der Schneebewegung zählt auch das zumeist nur oberflächige Abschürfen der Vegetations-, Humus- und Bodenschichten zur Gruppe des Massenschurfs durch Schnee. Der Schnee kann Unebenheiten bis zu einem Meter abschürfen (LAatsch \& GrotTenthaler 1972, S. 330), wobei die obersten Schichten allmählich ausgeschabt werden. Es handelt sich also nicht um einen Versatz ganzer Materialschollen oder einen Rutschungsprozess (SCHAuer 1975, S. 1). Es ist anzunehmen, dass der Großteil der sogenannten Schneeschurfblaiken dieser Prozessdynamik zuzuschreiben ist. 
Ebenso wie der Schneeschurf gehört der Narbenversatz durch Weidetiere in die Gruppe des Massenschurfs. An beweideten Hängen mit einer hohen Bestoßung bilden sich mit der Zeit parallel verlaufende Viehtrittwege (Viehgangeln). Auf ihnen kann es - besonders im steileren Randbereich - durch die Schubwirkung der Tierhufe zum Herauslösen von Teilen der Vegetationsdecke und des Bodens kommen (LAATSCH \& Grottenthaler 1972). Auch solche Stellen können Ansatzpunkte für weiteren Materialabtrag werden (vgl. Abb. 2).

Über die Bedeutung einer präformierten Inhomogenitätsfläche bei Vorgängen des Massenschurfs gibt es unterschiedliche Ansichten. STAHR (1997, S. 29) konnte nachweisen, dass der schollenförmige Bodenabtrag durchwegs entlang von Schichtwechseln erfolgt, unabhängig von Durchwurzelungstiefe und pedogener Entwicklung. Andere Arbeiten, vor allem solche, bei denen der oberflächliche Schneeschurf als Ursache der flachgründigen Denudation angesehen wird, nehmen keinen Bezug auf das Vorhandensein präformierter Gleitflächen, schließen sie allerdings auch nicht aus (BLECHSCHMIDT 1990, Kelch et al. 1977, Mössmer 1984, 1985, Stocker 2005). Gleiches gilt in Bezug auf den Materialversatz durch Viehtritt (LAatsch \& Grottenthaler 1972, S. 331), denn beide Abtragungsprozesse werden durch bewegende Medien ausgelöst und sind somit nicht auf Inhomogenitäten im Untergrund angewiesen.

\subsection{Sekundäre Weiterformung}

Die durch die beschriebenen Denudationsprozesse offen gelegten Flächen sind weiterem Abtrag durch Wind, Wasser und Schnee ausgesetzt, sodass sie in ihrer vertikalen und horizontalen Ausdehnung vergrößert werden. Auch die in Gebirgsräumen durch häufige Frostwechsel stark ausgeprägten frostdynamischen Prozesse spielen dabei eine Rolle, denn auch die Kriechbewegungen im Lockermaterial tragen zum Materialversatz an der Geländeoberfläche bei (AHNERT 1999, S. 135). Der Abtrag in vertikaler Richtung kann durch die Wirkung von Spaltenfrost auch in das anstehende Gestein fortschreiten (LaAtsch \& Grottenthaler 1973). Durch die Destabilisierung des Hanges setzt sich zudem häufig der Materialversatz hangaufwärts fort. Im Rahmen mehrerer Arbeiten wurden die Veränderungen der Abtragsflächen mithilfe vergleichender

\begin{tabular}{|l|c|c|c|c|c|c|c|c|}
\hline & \multicolumn{4}{|c|}{ Massenbewegung } & \multicolumn{4}{c|}{ Massenschurf } \\
\cline { 2 - 10 } & Schnee & Grav. & Wasser & Vieh & Schnee & Grav. & Wasser & Vieh \\
\hline Initialisierung & +++ & + & + & ++ & - & - & - & - \\
\hline Materialtransport & + & +++ & ++ & + & +++ & + & + & ++ \\
\hline sek. Erweiterung & + & + & +++ & + & ++ & + & +++ & ++ \\
\hline
\end{tabular}

+++ groß; ++ mittel; + gering; - nicht vorhanden; Grav. = Gravitation, sek. = sekundäre

Tab. 2: Überblick zu den steuernden Faktoren der Blaikenbildung und ihrer Bedeutung hinsichtlich der Teilprozesse (Grundlage: 13 Detailstudien aus dem Alpenraum, vgl. Tab. 3) 


\begin{tabular}{|c|c|c|c|c|c|c|c|c|c|c|c|c|c|}
\hline 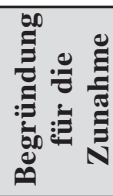 & & 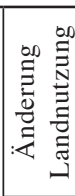 & 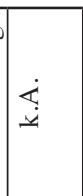 & 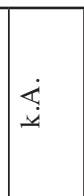 & & 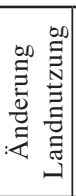 & 於 & 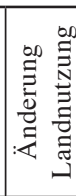 & 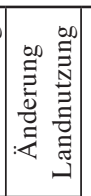 & & 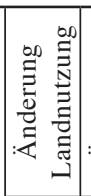 & 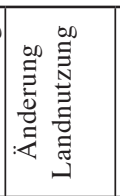 & 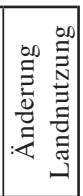 \\
\hline 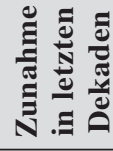 & & $\therefore$ & $\underset{\unlhd}{\unlhd}$ & $\mid \underset{\longleftarrow}{\check{\Perp}}$ & & $\stackrel{\cong}{\simeq}$ & $\cong \mathscr{0}$ & $\cong$ &.$\approx$ & & $\because \mathscr{\sim}$ & $\because \cong$ & $\therefore$ \\
\hline 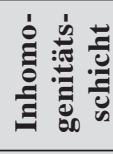 & & 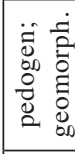 & 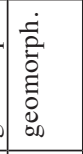 & 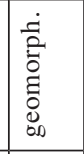 & & ذئذ & 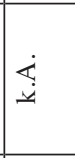 & $\underset{\perp}{\perp}$ & 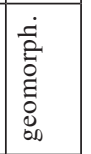 & & 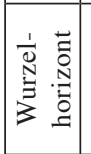 & 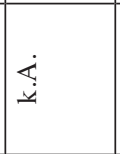 & 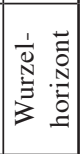 \\
\hline$\stackrel{\Xi}{\cong} \Xi$ & & $\begin{array}{l}3 \\
\text { ra } \\
0 \\
0\end{array}$ & $\begin{array}{l}\overrightarrow{1} \\
\dot{2} \\
\dot{Q}\end{array}$ & $\begin{array}{l}\vec{r} \\
\dot{2} \\
\dot{Q}\end{array}$ & & ذئ & $\underset{I}{\sharp}$ & $\underset{\Perp}{\check{I}}$ & 迎 & & 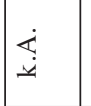 & $\underset{\Xi}{ \pm}$ & 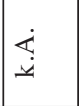 \\
\hline 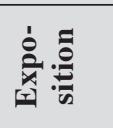 & & $\dot{\square}$ & $\stackrel{\searrow}{\Perp}$ & 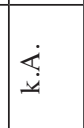 & & 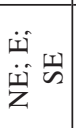 & \begin{tabular}{|l}
$\underline{y}$ \\
$\ddot{y}$ \\
$\ddot{z}$ \\
$\ddot{z}$
\end{tabular} & $\begin{array}{l}n \\
\ddot{y} \\
z \\
\ddot{z} \\
\ddot{z}\end{array}$ & $\begin{array}{l}z \\
z \\
z \\
\text { un }\end{array}$ & & $\begin{array}{l}3 \\
n \\
\ddot{n}\end{array}$ & os & 迎 \\
\hline 照 & & $\frac{y}{d}$ & 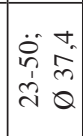 & 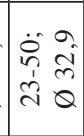 & & ले & $\begin{array}{l}n \\
q \\
\infty \\
\infty \\
n\end{array}$ & i & $\mid \begin{array}{l}n \\
y \\
\infty \\
m \\
n\end{array}$ & & $\begin{array}{l}\mathfrak{n} \\
\hat{d} \\
\hat{\sim}\end{array}$ & $\begin{array}{l}q \\
q \\
\dot{c} \\
m \\
m\end{array}$ & p \\
\hline 总 & & $\dot{\square}$ & $\underset{\longleftarrow}{\check{\leftrightarrows}}$ & $\mid \underset{\beth}{ \pm}$ & & 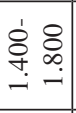 & 㝘 & 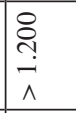 & 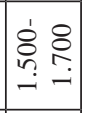 & & 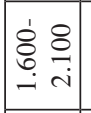 & 字 & {$\left[\begin{array}{cc}0 & 0 \\
0 & 0 \\
& 0 \\
- & -1\end{array}\right.$} \\
\hline 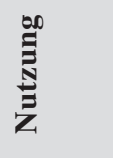 & & $\begin{array}{l}\frac{0}{0} \\
\frac{0}{0} \\
\frac{3}{0} \\
\frac{3}{3} \\
3\end{array}$ & 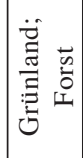 & 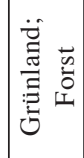 & & $\frac{\pi}{0}$ & 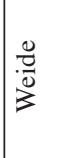 & 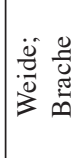 & $\frac{\pi}{0}$ & & 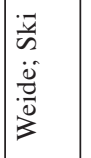 & 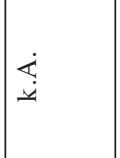 & 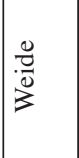 \\
\hline 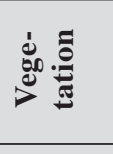 & & $\frac{\pi}{3 \pi}$ & 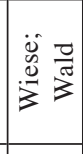 & 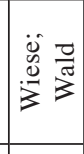 & & 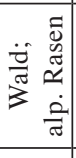 & 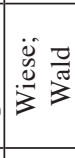 & $\dot{\Xi}$ & 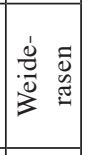 & 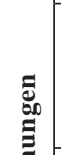 & $\begin{array}{l}0 \\
0 \\
0 \\
3 \\
3\end{array}$ & 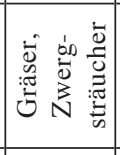 & 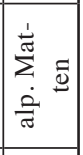 \\
\hline 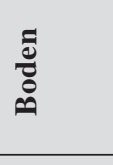 & & $\underset{亡}{\dot{\Delta}}$ & 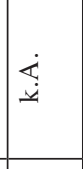 & $\underset{ \pm}{ \pm}$ & $\breve{\Xi}$ & 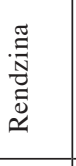 & 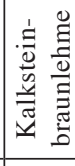 & $\dot{4}$ & 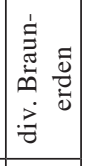 & 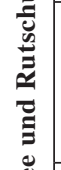 & 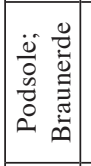 & 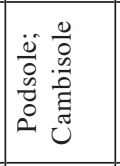 & 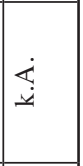 \\
\hline 鹃 & 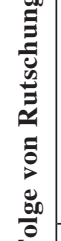 & 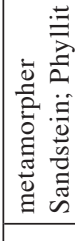 & 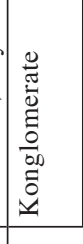 & 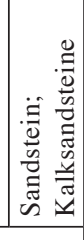 & 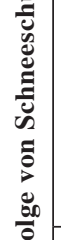 & 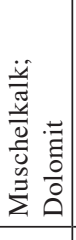 & 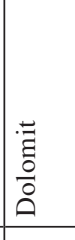 & 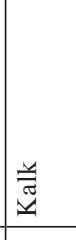 & 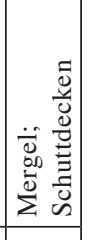 & 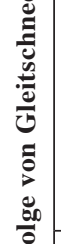 & 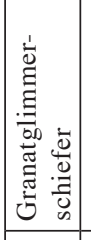 & 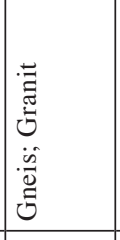 & 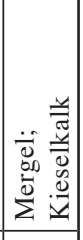 \\
\hline อ & 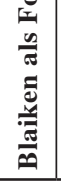 & 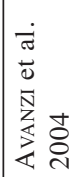 & 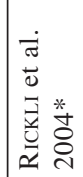 & & 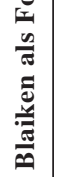 & 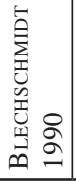 & 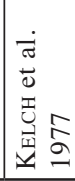 & 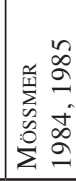 & 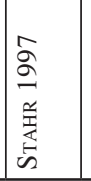 & 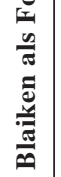 & 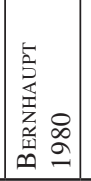 & 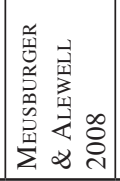 & 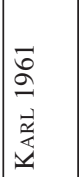 \\
\hline
\end{tabular}




\begin{tabular}{|c|c|c|c|c|c|c|c|}
\hline 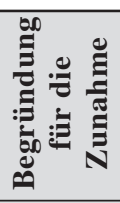 & & & 总 & 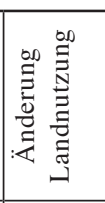 & 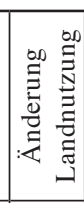 & 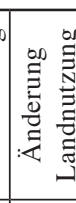 & $\Xi$ \\
\hline 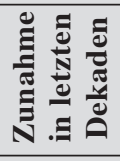 & & & $\stackrel{\Xi}{.}$ & $\stackrel{\Xi}{.}$ &.$\stackrel{\Xi}{\sim}$ & $\stackrel{\simeq}{\sim}$ & \\
\hline 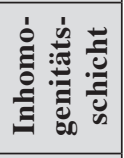 & & & 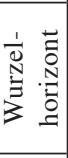 & 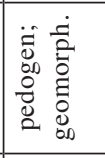 & 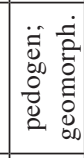 & $\dot{\square}$ & d \\
\hline 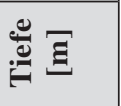 & 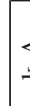 & & $\begin{array}{l}\text { cे ? } \\
0 \\
0\end{array}$ & 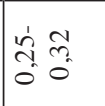 & 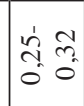 & 它 & 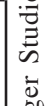 \\
\hline 商高 & 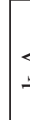 & & $\stackrel{\unlhd}{\unlhd}$ & 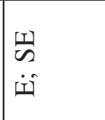 & $\begin{array}{l}\text { 岀 } \\
\text { if }\end{array}$ & $\begin{array}{l}\ddot{3} \\
\ddot{3} \\
\ddot{n}\end{array}$ & 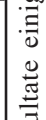 \\
\hline 矛 & & & 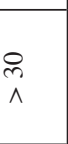 & $\begin{array}{l}\text { fo } \\
\text { d } \\
\text { d }\end{array}$ & 古 & 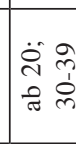 & : \\
\hline$:$ & & \&) & 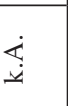 & 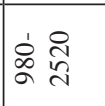 & 字导 & 莖 : & 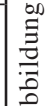 \\
\hline 些 & & & 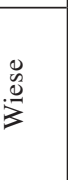 & 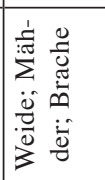 & 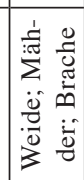 & 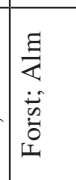 & $\frac{3}{2}$ \\
\hline 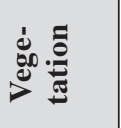 & 7 & & 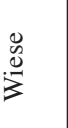 & 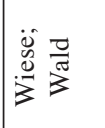 & $\begin{array}{l}\ddot{y} \\
0 \\
3 \\
3 \\
3\end{array}$ & 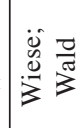 & \\
\hline فّㅠ. & & 离 & 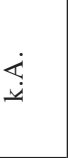 & 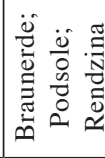 & 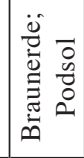 & $\stackrel{\square}{\longleftarrow}$ & \\
\hline كै & 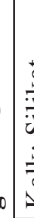 & & 羔 & 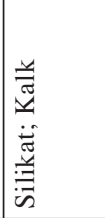 & 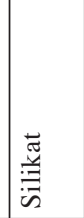 & 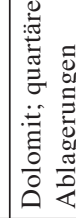 & \\
\hline$\stackrel{\Xi}{=}$ & & & 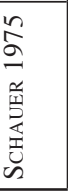 & 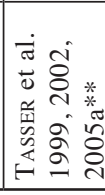 & & 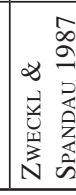 & \\
\hline
\end{tabular}




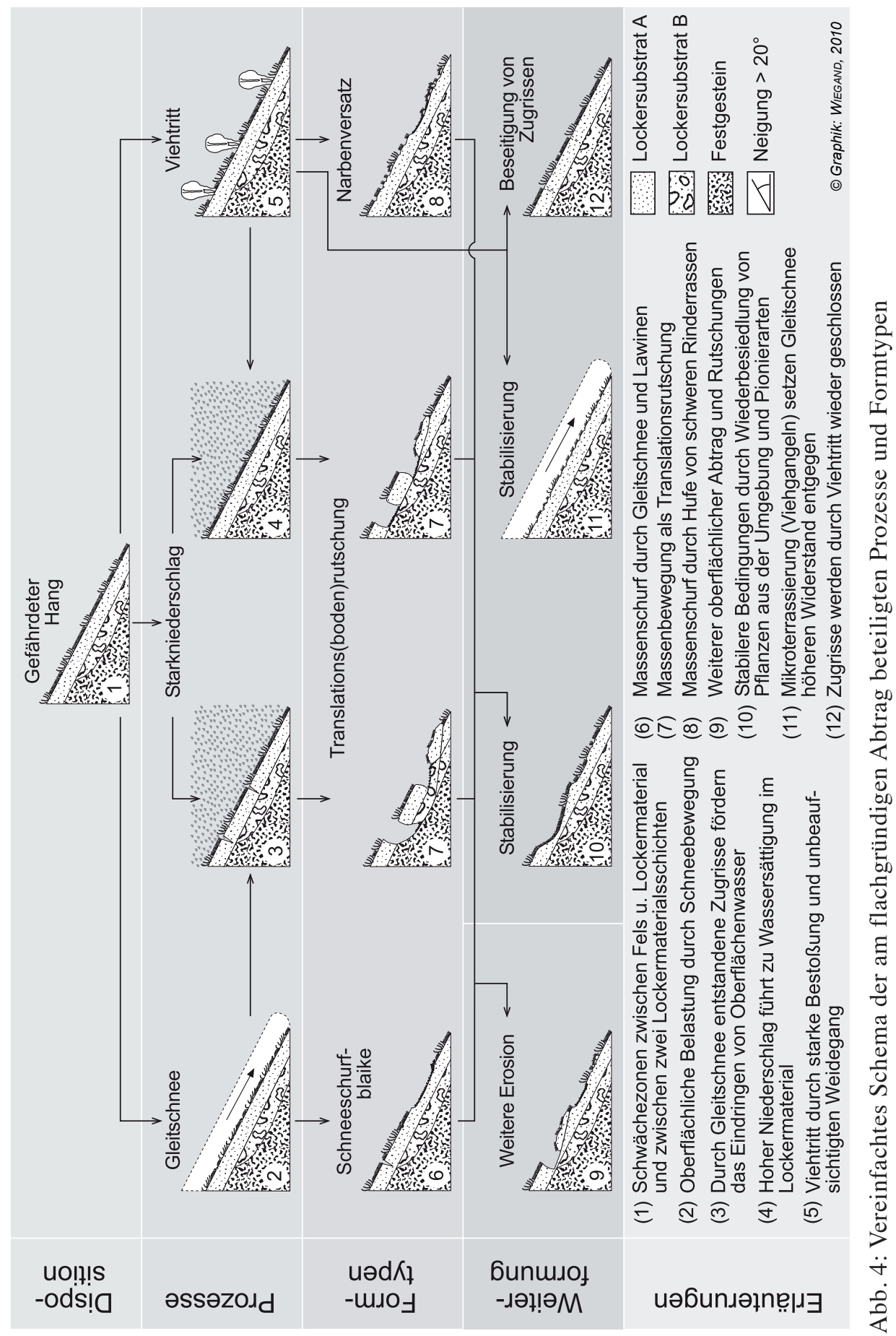


Luftbildauswertungen über bestimmte Zeiträume untersucht. In den meisten Fällen konnte eine Zunahme festgestellt werden, wobei diese jedoch sowohl eine Ausweitung bereits bestehender Flächen als auch die Entstehung neuer Denudationsflächen beinhaltet (Bernhaupt 1980, Blechschmidt 1990, Kelch et al. 1977, Meusburger \& Alewell 2008), sodass kaum eindeutige Aussagen zum Ausmaß der sekundären Weiterformung vorliegen. In einigen Fällen gelang es der Vegetation, sich auf den Erosionsflächen erneut anzusiedeln. SCHAUER (1975, S. 9) beschreibt das Aufkommen von Arten einerseits aus der intakten Umgebung, andererseits von Pionierarten wie Tussilago farfara L., was zum Vernarben der Denudationsflächen und einem - je nach Sukzessionsstadium - zunehmenden Schutz vor weiteren Abtragsprozessen führt.

Die unterschiedlichen Auffassungen zu den beteiligten Prozessen lassen sich insofern zusammenfassen, als es sich bei der angeführten Denudationserscheinung (Blaike) entweder um eine Massenbewegung in Form einer Translationsrutschung oder um Massenschurf in Form von Schneeschurf oder Viehtritt handelt. Daher wird im Folgenden begrifflich nach flachgründigen Rutschungen auf der einen und Massenschurf durch Schnee und Viehtritt auf der anderen Seite unterschieden. Der Terminus Blaike findet nur noch dann Verwendung, wenn er auf die undifferenzierte Gesamtheit dieser Formen hindeuten soll.

\section{Steuernde Faktoren für flachgründige Massenbewegungen und Massenschurf}

Es gibt eine Reihe von Faktoren, die bei der Entstehung flachgründiger Denudation eine Rolle spielen. Ihr spezifischer Anteil ist im Einzelfall oft schwer abzuschätzen, da die Kombination der relevanten Faktoren stets eine individuelle ist. Dennoch sollen die einzelnen Einflussparameter eingehender charakterisiert und diskutiert werden.

\subsection{Gestein}

Die geologischen Rahmenbedingungen haben einen deutlichen Einfluss auf die flachgründige Abtragung. Die unterschiedlichen lithologisch-morphologischen Eigenschaften der diversen Ausgangsgesteine prägen nicht nur das Relief, sie stellen auch das Material für die Lockergesteinsdecken und für die Bodenbildung bereit, welches sowohl die Bodentypen als auch deren physikalisch-chemischen Eigenschaften mitbestimmt.

Flachgründiger Abtrag bildet sich im Lockermaterial aus, kann sich aber auch bis zum anstehenden Gestein fortsetzen (STOCKeR 1985, 2005). Mit Erreichen des Festgesteins herrschen dann andere Prozessabläufe vor. Das Lockermaterial ist in der Regel im oberflächennahen Bereich verwittert und von Bodenbildung überprägt. Untersuchungen aus geologisch sehr verschieden aufgebauten Gebieten belegen, dass es auf sehr unterschiedlichen Gesteinen zur Ausbildung von flachgründigem Hangabtrag kommen kann. Die Zusammenhänge sind zudem eher indirekter Natur und wirken über die Geländeformen und die minerologische Zusammensetzung des Lockermaterials. 
Der Aufbau des Lockermaterials in Form von Regolith- oder Schuttdecken ist in Bezug auf deren Stabilität von großer Bedeutung. Als wichtige Merkmale wären die Mehrschichtigkeit sowie die Lagerungsdichte und Korngrößenzusammensetzung zu nennen (VEIT 2002, S. 128 ff.; VeIT et al. 2002). STAHR (1997) konnte beispielsweise nachweisen, dass die hangenden Schichten der Schuttdecken lockerer gelagert sind und einen niedrigeren Skelett- und höheren Schluffgehalt aufweisen als das liegende Lockermaterial.

Wie groß der Einfluss der Zusammensetzung des Lockermaterials auf den flachgründigen Versatz ist, konnte noch nicht eindeutig geklärt werden. In der Regel handelt es sich um Hangschutt, wobei die Prozesse sowohl auf kalkreichem als auch auf silikatischem Lockermaterial zu belegen sind. Innerhalb einzelner Untersuchungsgebiete lassen sich dennoch Häufungen auf bestimmten Gesteinen feststellen. So konnten Avanzi et al. (2004) ein vermehrtes Auftreten auf Sandstein und Phyllit, Kelch et al. (1977) und Mössmer (1985) auf Kalk und Dolomit beobachten. Es ist davon auszugehen, dass die Korngrößenzusammensetzung des Substrates entscheidender ist als die mineralische Zusammensetzung, da erstere den Wasserhaushalt maßgeblich steuert. Diesbezüglich aber fehlt es an vergleichenden Studien, die auch dadurch erschwert werden, dass es in den meisten Untersuchungsgebieten an räumlich und inhaltlich differenzierten Substratdaten mangelt (z.B. RiCKLI et al. 2004, S. 45).

\subsection{Lageeigenschaften und Relief}

Als wirksame geomorphologisch-topographische Parameter werden - in der Reihenfolge ihrer Bedeutung - die Hangneigung, die Exposition, die Höhe über dem Meer sowie die Rauhigkeit des Untergrunds ins Feld geführt. Die Hangneigung gilt als wichtigster geomorphologischer Parameter, da nur auf Flächen ab einem gewissen Neigungswert Massen in Bewegung geraten. Aus den bisherigen Studien ergeben sich für die durch Schneeschurf bedingte Blaikendenudation eine mittlere minimale Hangneigung von $31^{\circ}$ und eine mittlere maximale Hangneigung von $41^{\circ}$ (vgl. Tab. 3), wobei unter anderem Bernhaupt (1980), Mössmer (1985) und Zweckl \& Spandau (1987) Schurfaktivitäten bereits ab 20 bis $26^{\circ}$ Neigung beobachten konnten. Für Blaiken als Folge von Rutschungen kann eine Neigungsspanne von 23 bis $45^{\circ}$ angegeben werden (vgl. Tab. 3), wobei $82 \%$ der Rutschungen in den Schweizer Untersuchungsgebieten bei $>30^{\circ}$ Neigung stattfanden (RICKLI et al. 2004, S. 43). Ab Neigungen über $45^{\circ}$ wird eine Abnahme der Blaikenhäufigkeit verzeichnet. Dies lässt sich dadurch erklären, dass sich in so steilen Bereichen nur in Ausnahmefällen unverfestigte Lockersedimente halten sowie Boden und Vegetationsdecke entwickeln können (BLECHSCHMIDT 1990, TASSER et al. 2005b).

Auch die Exposition der Hänge scheint eine Rolle zu spielen, wobei sich jedoch anhand der durchgeführten Arbeiten bisher kein eindeutiges Bild abzeichnet. In einigen Untersuchungsgebieten tritt der flachgründige Abtrag vor allem an SW- bis NE-exponierten Hängen auf (Meusburger \& Alewell 2008, Bernhaupt 1980, BlechSCHмIDT 1990). In anderen Studien wurden die Denudationsstellen vermehrt auf N- bis NW- (Kelch et al. 1977, Mössmer 1984, Stahr 1997) bzw. NE- bis SE-exponierten 
Hängen festgestellt (TASSER et al. 2005b). Zum Teil mögen sich in solchen Auswertungen auch die Talausrichtung und geologisch-morphologische sowie nutzungsbedingte Unterschiede niederschlagen.

Unabhängig davon wirken sich die Expositionsunterschiede auf den lokalen Wasserhaushalt aus, der vor allem durch die Dauer der Sonneneinstrahlung, die Verdunstung und die Schneeschmelze gesteuert wird. Die von TASSER et al. (2005b) sowohl im Stubaital als auch im Passeiertal festgestellte Häufung von Versatzstellen auf NE- bis SE-exponierten Hängen mag mit diesem geringeren Energieinput und der stärkeren Durchfeuchtung - vor allem nach der Schneeschmelze - zusammenhängen. LAATSCH \& Grottenthaler (1973) wie auch TAsser et al. (2005b) weisen zudem darauf hin, dass die erhöhte Einstrahlung an SE-exponierten Hängen die Bildung von Gleitschnee und damit beide Formen der Blaikenbildung begünstigt. Die Exposition modifiziert also in komplexer Weise die anderen Faktoren und damit die ablaufenden Prozesse.

Die vertikalen Temperatur- und Niederschlagsgradienten führen dazu, dass auch der Höhe ein wesentlicher, wenngleich nur indirekter Einfluss auf den Wasserhaushalt und damit auf Verwitterung und Bodenbildung zukommt. Am wichtigsten in diesem Zusammenhang scheint jedoch die Schneesituation zu sein, da Schneegleiten und Schneeschurf an der Bildung flachgründiger Denudation wesentlich beteiligt sind. In diesem Zusammenhang weist Mössmer (1985, S. 106) auf den engen statistischen Zusammenhang zwischen Meereshöhe und schurfbedingter Blaikendenudation hin und begründet ihn durch die zunehmende Mächtigkeit der Schneedecke mit ansteigender Höhe sowie mit den spezifischen Reliefverhältnissen in der entsprechenden Höhenstufe. Aus den Auswertungen der bisherigen Studien ergibt sich eine Mindesthöhe von $1.000 \mathrm{~m}$, wobei sich der Großteil der Abtragsflächen zwischen 1.200 und $2.500 \mathrm{~m}$ über dem Meer bildet (vgl. Tab. 3). Diese Höhenangaben entsprechen weitgehend denen des verstärkten Schneegleitens. So konnten LAATSCH \& BAum (1976, S. 331) für die Bayerischen Alpen intensiven Schneeschurf ab einer Höhe von 1.200 m feststellen. Für den Schweizer Alpenraum wird das Auftreten von Schneegleiten zwischen 1.200 bis 2.500 m beschrieben (IN DER GAND 1972, zitiert nach Mössmer 1985, S. 106). Das von Mössmer (1985, S. 106) angegebene Maximum der Denudationsflächen auf aufgelassenen Wiesen zwischen 1.200 und 1.400 m beschränkt sich auf Schattenhänge, da hier der Schnee im Frühjahr länger liegen bleibt, und es somit zum Schneeschurf kommt. Auf diese Weise wird der Faktor Höhe durch den Faktor Exposition überlagert. Bei Rutschungen, hervorgerufen durch starke Niederschläge, ließ sich für Schweizer Untersuchungsgebiete ebenfalls ein Zusammenhang zwischen der Höhe und der Rutschungsaktivität feststellen, denn sowohl die intensivsten Niederschläge als auch die meisten Rutschungen fanden in mittleren Höhenlagen statt (RICKLI et al. 2002, S. 441). Hier wird die Höhenlage durch die Niederschlagsintensität - also indirekt - für die Abtragsdynamik wirksam.

Nicht nur die Lageeigenschaften, sondern auch die konkrete Reliefformung bestimmt die Entstehung flachgründiger Denudationsflächen. So wird durch die Rauhigkeit - z.B. durch die Mikroterrassen der Viehgangeln - den Schneemassen ein erhöhter Widerstand entgegengesetzt, sodass trotz entsprechender Hangneigungen Schneeschurf und die Bildung von Zugrissen ausbleiben (Blechschmidt 1990, S. 44; Schauer 1975, S. 18) (vgl. Abb. 4). Auch das gesamte Hangprofil hat einen Einfluss. So besitzt laut 
Mössmer (1985, S. 76) die Ausdehnung des Hanges oberhalb der Denudationsfläche einen erheblichen Einfluss auf deren Entstehung, was mit der verfügbaren Menge an Hangwasser zusammenhängen dürfte.

\subsection{Boden}

Ein großer Teil der im Alpenraum vorkommenden Blaiken bildet sich in den oberen Dezimetern von Lockersedimenten und somit im Tiefenbereich der Bodenbildung (vgl. Tab. 3). Daher ist die Annahme naheliegend, dass dem Boden dabei auch eine spezifische Bedeutung zukommt. In erster Linie sind die bodenphysikalischen Eigenschaften wie Korngrößenverteilung, Skelettgehalt, Gefüge, Dichte und Porenverteilung relevant. Sie entscheiden darüber, wie groß die Kapazitäten des Bodens für Infiltration und Wasserspeicherung sind, die Abfluss- und Abtragsgeschehen mitbestimmen (LÖHMANNSRÖBEN 2002). Aber auch der Bodenchemismus, gekennzeichnet unter anderem durch Kalkgehalt, pH-Wert sowie Anteil, Verteilung und Qualität der organischen Substanz, steuert die Stabilität von Böden. Der Humus beispielsweise beeinflusst die Aggregatbildung und den Wasserhaushalt entscheidend (KELCH et al. 1977), der pH-Wert die Stoffverlagerung innerhalb des Profils, die zur Bildung von stauenden Horizonten führen kann, sowie die Aktivität des Edaphons - d.h. der Bodenorganismen - und damit das Ausmaß der Bioturbation und aggregatstabilisierenden Ton-HumusKoppelung.

In den unterschiedlichen Fallstudien werden die jeweiligen Bodentypen beschrieben, in denen der flachgründige Abtrag stattfindet: Auf kalkdominiertem Substrat handelt es sich zumeist um Rendzinen (BLECHSCHMidt 1990), auf intermediärem und silikatischem Substrat der Zentralalpen um Braunerden und Podsole (Meusburger \& Alewell 2008, TAsser et al. 2005b). Ein engerer Zusammenhang von Bodentyp oder Subtypen und dem Entstehen von Blaiken lässt sich nicht belegen. Selbst im Hinblick auf die Bodeneigenschaften konnten EidT \& LöHMANnsRöBen (1996) keine Korrelation feststellen. In ihrem Fall waren die Parameter Hangneigung, Exposition und Vegetation ausschlaggebender. In anderen Studien ergeben sich jedoch deutliche Hinweise auf den Einfluss bestimmter Bodenparameter. Untersuchungen aus den Nördlichen Kalkalpen beispielsweise betonen den hohen Schluffgehalt, durch den die Böden durch das in die Zugrisse eindringende Wasser zum Zerfließen neigen und rasch einen hohen Porenwasserdruck aufbauen. Die abgleitenden Rasenschollen lagen im Falle dieser Untersuchung auf einer zu Brei zerflossenen Schicht (SCHAUER 1975, S. 17). Gegensätzliche Befunde liefert StAHr (1997, S. 110, 117) aus dem Nationalpark Berchtesgaden, wo sich schluffreiche Böden trotz starker Niederschläge als stabil erwiesen. Auch die Mächtigkeit von Böden konnte mit dem Auftreten von flachgründigen Rutschungen in Verbindung gebracht werden, wobei tiefgründigere Böden durch ihre Wasseraufnahmekapazität anfälliger gegenüber Rutschungen zu sein scheinen als flachgründigere (TASSER et al. 2005b, S. 207).

Die im Hinblick auf die Hangstabilität sehr bedeutsamen Inhomogenitätsflächen entstehen teilweise erst im Zuge der Bodengenese. Es bilden sich Bodenhorizonte mit unterschiedlichen physikalischen Merkmalen, z.B. durch die Prozesse der Verlehmung, 
Tonverlagerung und Podsolierung, durch die sich stauende Horizonte im Unterboden herausbilden können. Aber auch bei weniger entwickelten Böden wie Rendzina oder Regosol stellt die Grenze zwischen A- und C-Horizont oft einen relativ abrupten Wechsel einiger Bodenmerkmale wie organische Substanz, Durchwurzelung, Skelettgehalt und Korngrößenverteilung dar. Solch eine akzentuierte Horizontierung bedeutet häufig eine gewisse Destabilisierung.

\subsection{Vegetation}

Die Vegetation der Kulturlandschaften im Alpenraum ist das Produkt Jahrhunderte langer Bewirtschaftung. Sie steht also in enger Verbindung mit der jeweiligen Landnutzung. Zusätzlich entscheiden die Bodeneigenschaften darüber, welche Pflanzen sich ansiedeln können, vor allem Gründigkeit, Wasser- und Nährstoffhaushalt und $\mathrm{pH}$-Wert. Doch werden wichtige chemische Bodeneigenschaften über den Streuanfall der Pflanzen mitbestimmt (pH-Wert, C/N-Verhältnisse, Humusqualität), was sich auch auf das Bodengefüge und den Stofftransport innerhalb der Böden auswirkt (TASSER et al. 1999). Auch der Grad der Durchwurzelung (Bau der Wurzeln, Durchwurzelungsdichte, Durchwurzelungstiefe) beeinflusst den Zusammenhalt des Bodenmaterials erheblich. So bieten die Wurzeln von Bäumen nur dann einen mechanischen Schutz vor Rutschungen, wenn sie bis unter die Gleitfläche reichen (RICKLI et al. 2002, S. 438). Auf die Bildung einer Imhomogenitätsschicht entlang einheitlicher Wurzelhorizonte (SCHAuER 1975) wurde bereits hingewiesen.

Die naturnahe Vegetation der montanen Höhestufen wäre - zumindest außerhalb der Zentralalpen - ein strukturreicher Bergmischwald, in der Höhe abgelöst von Nadelwald, der sich schließlich bis zur Waldgrenze erstreckt. Im Hinblick auf eine natürliche und dauerhafte Erosionsvermeidung wären gesunde, naturnahe Wälder besonders günstig (Hamberger \& Moser 2008, Rickli et al. 2002, Schauer 1999), zumal diese eine optimale Ausnutzung des potenziellen Wurzelraums bieten (WITZIG et al. 2004). Die Hänge erhalten durch den Zusammenhalt der Wurzeln eine hohe Stabilität, die kinetische Energie des Niederschlags wird durch den Bewuchs gedämpft und ein Teil des Wassers wird durch Transpirations- und Interzeptionsvorgänge zurückgehalten, wodurch Hochwasserspitzen gekappt werden können (BunzA et al. 1996). Außerdem sind unter Wald niedrigere Ausgangsporenwasserdrücke zu verzeichnen, was sich in Bezug auf Starkniederschläge bis zu einem gewissen Grad positiv auswirkt (RICKLI et al. 2002, S. 438). Auch die Bewegung der Schneedecke und damit ihre Denudationsleistung vermindern sich durch das Vorhandensein von widerstandsfähigem Bewuchs erheblich. Die subalpine Höhenstufe, oft verstanden als Übergangssaum zwischen Wald- und Baumgrenze (Ellenberg 1996, S. 562), ist von Sträuchern wie Pinus mugo L. und Alnus viridis (Chaix) DC. und zahlreichen Zwergsträuchern gekennzeichnet (Reisigl \& Keller 1994). Innerhalb dieser Höhenstufe bilden diese einen Schutz vor Abtragung durch Schneeschurf (SCHAUER 1999).

Auf einem großen Gebietsanteil des ehemaligen Waldes finden sich heute Grünlandflächen mit - je nach Art und Intensität der Nutzung - unterschiedlichem Besatz an Gräsern und Kräutern. In den Nordalpen konnte auf waldfreien Hängen mit einer 
Dominanz an ertragsreichen Pflanzen mit hohem Anteil oberirdischer Phytomasse wie Geranium sylvaticum L., Trifolium pratense L., Anthoxanthum odoratum L., Alchemilla vulgaris L., Festuca violacea L. und Dactylis glomerata L. eine verstärkte Bereitschaft zur Abtragung verzeichnet werden. Auf Flächen mit einem geringeren Auftreten von Denudationserscheinungen fanden sich hingegen weniger ertragsreiche Arten, bei denen der Anteil an oberirdischer Phytomasse geringer ist als der der unterirdischen, wie Anthyllis alpestris L., Lotus corniculatus L., Carduus defloratus L., Ligusticum mutellina (L.) Crantz und Plantago alpina L. (SCHAUER 1975, S. 14). Demnach spielt das Verhältnis von ober- zu unterirdischer Biomasse wohl in zweierlei Hinsicht eine wichtige Rolle, erstens, was den Zusammenhalt im Boden, zweitens, was die Angriffsmöglichkeiten für den Gleitschnee betrifft.

Wenn die Bewirtschaftung von Wiesen oder Weiden aufgegeben wird, kann sich durch Sukzession allmählich erneut eine quasinatürliche Waldvegetation entwickeln (MAAG et al. 2001, TASSER \& TAPPEINER 2002), die weitgehenden Schutz vor Erosion bieten kann. Wie effizient der Schutz wirklich ist, hängt nach RickLI \& Graf (2009, S. 41) allerdings stark vom Zustand des Bestandes ab und muss differenzierter betrachtet werden.

\subsection{Nutzung}

Durch Rodung und landwirtschaftliche Inwertsetzung der Flächen kommt es nicht nur zu mehr mechanischem Bodenabtrag, sondern auch zur Reduktion der organischen Substanz. Dieser sogenannte Humusschwund trägt nach Kelch et al. (1977) durch die Abnahme der Ton-Humus-Koppelung zur Stabilitätsminderung bei. Dolomit- und Kalksteinhänge weisen einen erhöhten Verlust von Humus auf, da dieser aufgrund des geringen Tongehaltes weniger stark gebunden werden kann. Nach LAATSCH \& Grottenthaler (1973) führt dies nicht nur zur Abnahme der Stabilität, sondern auch des Wasserhaltevermögens.

Die traditionelle Form extensiver Weide- und Wiesenwirtschaft schloss eine arbeitsintensive Pflege der Flächen ein. Durch das Putzen und Schwenden, also das Entfernen von Steinen und Hölzern sowie das Abbrennen vereinzelt aufkommender Sträucher und das Beseitigen bereits entstandener Schäden konnten die Flächen weitestgehend vor Erosionsangriff bewahrt werden (Bernhaupt 1980, BleChSCHMidT 1990). Auf den traditionell genutzten Flächen findet sich zudem eine hohe pflanzliche Diversität (MAAG et al. 2001, VEIT 2002) mit hohem Durchwurzelungsgrad und damit stabilisierender Wirkung (TASSER et al. 2005b).

Auf Grundlage von Untersuchungen in 23 Wildbacheinzugsgebieten am bayerischen Alpennordrand konnten von Bunza et al. (1996) Translationsrutschungen mit nutzungsbedingten Bodenveränderungen in Verbindung gebracht werden. Auch in der Arbeit von EIDT \& LöHMANNSRÖBEn (1996, S. 253) heben sich durch Translationsrutschungen betroffene Flächen von anderen durch hohe Anteile nutzungsbedingter Bodenveränderungen - besonders durch Weidenutzung - ab. Die Folgen treten vor allem durch die Entstehung von Weidestausohlen, Trittverletzungen der Grasnarbe und der Humusschicht in Erscheinung. Ob jedoch ein kausaler Zusammenhang zwischen 
den Bodenveränderungen und den morphodynamischen Prozessen besteht oder beide nur Folgen des Nutzungs- und Vegetationswandels sind, konnte nicht geklärt werden.

Der sich in den letzten Dekaden in den Alpen vollziehende sozio-ökonomische Wandel führte zu durchgreifenden Änderungen traditioneller Nutzungsformen. Aufgrund geringer wirtschaftlicher Anreize und fehlender personeller Ressourcen ging vor allem die Almbewirtschaftung zurück (B̈̈TZING 2003, S. 225ff.). Das betraf vor allem schwer zugängliche und somit ökonomisch unrentable Flächen. In vielen Fällen geriet die Landwirtschaft zum Nebenerwerb (BERNHAupt 1980). Somit setzte nicht nur die Arbeit des Almputzers aus, auch der Weidebetrieb lief mehr und mehr unbeaufsichtigt ab, was zur Ausdehnung von Trittschäden beitrug (LAATSCH \& GROTTENTHALER 1973). Es kam aber neben der Extensivierung und dem Brachfallen der Flächen stellenweise auch zu Intensivierungen, d.h. in der Regel zu einer Zunahme der Bestoßung auf leicht zugänglichen und damit ökonomisch attraktiveren Nutzflächen (TASSER et al. 2005a). Beides muss in seinen Auswirkungen genauer betrachtet werden.

Durch Brachlegung ist ein rascher Wandel der Pflanzendecke zu beobachten. Dieser ist zumeist gekennzeichnet durch eine Abnahme der Biodiversität, indem sich dominante Arten auf Kosten weniger dominanter ausbreiten können (TASSER \& TAPPEINER 2002). Häufig dominieren langhalmige Gräser (Deschampsia caespitosa (L.) Beauv., Molinia arundinaceae Schrank., Carex sempervirens Vill.), die durch den Schnee auf den Boden gedrückt werden und dort eine glatte Fläche bilden, die dem Schnee nur minimalen Reibungswiderstand bietet und somit das Schneegleiten sehr begünstigt (LAATSCH \& GrotTenthaler 1973). Die Abnahme der Artenvielfalt trägt zur Bildung eines einheitlichen Wurzelhorizontes bei, was ebenso die flachgründige Denudation fördert. TASSER et al. (2005a) konnten zudem nachweisen, dass mit dem Aussetzen der Bewirtschaftung auch eine Verringerung der Durchwurzelungsdichte einhergeht. Dieser Effekt kann mit der Versauerung der Böden in Verbindung gebracht werden, die wiederum durch die Zunahme der Zwergsträucher mit entsprechender Streuakkumulation vorangetrieben wird. Mit diesen Veränderungen geht auch eine Verschiebung in der Artenzusammensetzung der Regenwürmer und damit der Bioturbationsleistung einher (SEeber et al. 2005). Im weiteren Sukzessionsverlauf kommen nach einigen Jahren die ersten Bäume auf, und mit der langsamen Verdichtung des Bestandes tritt wieder eine hohe Stabilität ein (KöRNER 1999, S. 292). In Abhängigkeit von verschiedenen Faktoren kann sich schon nach rund 20 Jahren ein mannshoher Bestand etabliert haben (MAAG et al. 2001, S. 10).

Während der Phase zwischen der Brachlegung und der Etablierung eines Waldbestandes steigt zunächst die Denudationsgefährdung für einige Jahre (TASSER et al. 2005b, S. 204). Dies bedeutet allerdings nicht, dass es zwangsläufig zu einer Zunahme von Abtragsereignissen kommen muss, es wird lediglich die Disposition verstärkt. Zusätzlich bedarf es eines auslösenden Moments in Form von Schneegleitbewegungen oder Starkniederschlägen (TASSER et al. 2005b).

Neben der im Alpenraum verbreiteten Extensivierung sind ebenso zunehmend intensivere Bewirtschaftungen zu beobachten, sei es durch eine höhere Bestoßungszahl auf Weideflächen (Bernhaupt 1980, Blechschmidt 1990) oder durch Düngung und eine Zunahme der Jahresschnitte bei Mähwiesen. Solche Nutzungsintensivierung ist oft an die Hofnähe oder eine gute Erreichbarkeit der Flächen mit landwirtschaftlichen 
Maschinen geknüpft (TASSER \& TAPPEINER 2002). Auch Bewirtschaftungsintensivierung führt in der Regel zur Abnahme der pflanzlichen Diversität (TASSER et al. 2005a), denn oft breiten sich vom Vieh gemiedene (v.a. Nardus stricta L.) oder durch Düngung besonders begünstigte Arten aus (z.B. Agrostis capillaris L., Festuca ruba L.) und verdrängen andere (Lichtenegger 1988, zitiert nach Veit 2002, S. 198). Eine hohe Bestoßungszahl kann bei unbeaufsichtigtem Weidegang zu verstärktem Bodenversatz durch Viehtritt führen (LAatsCH \& GrotTenthaler 1973). Andererseits konnte BleCHsснміDт (1990) an Testflächen im Karwendel belegen, dass schneeschurfbedingte Blaiken auf beweideten Flächen deutlich seltener als auf unbeweideten auftreten. Als mögliche Gründe hierfür führt die Autorin die Terrassierung der Hänge durch Viehgangeln sowie das Vorherrschen überwiegend kurzer Gräser auf (vgl. Abb. 4).

Es können also auch im Hinblick auf die Nutzung kaum pauschale Aussagen getroffen werden, denn die Auswirkungen hängen nicht nur von den naturräumlichen Rahmenbedingungen, sondern ebenso von Nutzungsintensität und begleitendem Pflegeaufwand ab (Blechschmidt 1990, Bernhaupt 1980, Riedl 1982). Dennoch kann STAHR (1997, S. 7) prinzipiell gefolgt werden, wenn er das vermehrte Auftreten von Blaiken als Folge und Indikator des wirtschaftlichen Wandels betrachtet.

\subsection{Klima}

Das Klima wirkt in mannigfaltiger Weise auf die Prozesse der Blaikenbildung. Allerdings finden sich dazu kaum differenzierte Angaben oder gar vergleichende Untersuchungen, was damit zusammenhängen dürfte, dass der Klimafaktor von den anderen Faktoren zu stark überlagert wird. Dennoch sind sowohl die jährlichen Niederschlagsmengen (Disposition) als auch die Häufigkeit von Extremereignissen (Auslösung) als relevant einzuschätzen, ebenso die Mächtigkeit und der Aufbau der Schneedecke. Entsprechend findet man für jede Region gutachterliche Einschätzungen, die den Zusammenhang von Niederschlag und Blaikenbildung betreffen. Um diese - in einem mittleren Maßstabsbereich - überprüfen zu können, wäre ein wesentlich dichteres Netz an Klimastationen nötig. Großräumig jedoch bestätigen sich gewisse Tendenzen, beispielsweise, dass am niederschlagsreichen Nordalpenrand - bei kritischer Disposition der übrigen Einflussparameter - ein häufigeres Auftreten flachgründiger Rutschungen als in den relativ trockenen, zentralalpinen Lagen festgestellt werden kann. In letzteren bedarf es besonderer Extremereignisse und bestimmter, vor allem glimmerreicher Gesteine, um flachgründige Rutschungen in nennenswertem Maß auftreten zu lassen.

\subsection{Relevante Faktorenkombinationen und mögliche Gründe für die aktuelle Zunahme der flachgründigen Denudation in den Alpen}

Alle angeführten Studien bestätigen, dass die flachgründige Denudation nicht monokausal, sondern nur durch komplexe Verknüpfung verschiedener Einflussfaktoren zu erklären ist (z.B. EidT \& LöHMANNSRöBben 1996, HAMBERGER \& Moser 2008, TASSER 
et al. 2003), wobei die Anteile der Einzelparameter auf den Abtragsprozess kaum abzuschätzen sind. Daher sind die relevanten Prozesse kaum über eindeutige Schwellenwerte eingrenzbar. Damit es zum Abrutschen einer Bodenscholle kommt, müssen in der Regel mehrere Parameter im kritischen Bereich liegen. Wird beispielsweise nur die kritische Hangneigung überschritten, muss es zu keinem Materialversatz kommen, wenn die übrigen Einflussparameter Stabilität gewährleisten. Die Verschiebung eines variablen Faktors kann allerdings gravierende Änderungen im System bewirken, sodass zum Beispiel nutzungsbedingte Veränderungen vermehrten Abtrag auslösen können.

Nicht nur die Wirkung der Einzelfaktoren, sondern auch die Wirkung ihrer kritischen Kombinationen wird von den Autoren verschieden eingeschätzt. Das liegt sicher teilweise in den unterschiedlich ausgestatteten Untersuchungsgebieten begründet. Doch auch die jeweilige Blickrichtung der Disziplinen scheint für die Gewichtung der Einflussparameter eine gewisse Rolle zu spielen. Zudem muss berücksichtigt werden, auf welcher Maßstabsebene man das Phänomen Blaike untersucht: Vergleicht man die Denudationsflächen unterschiedlicher Testgebiete, treten eher die geologischen Unterschiede hervor. Bei großmaßstäblicherer Betrachtung - beispielsweise beim Vergleich von Rutschungsflächen innerhalb eines Tals oder an einem Hang - wird der Einfluss von Boden- und Vegetationsparametern deutlicher (TASSER et al. 1999). Die Analyse dieser Zusammenhänge wird dadurch zusätzlich erschwert, dass dort, wo eine Denudationsfläche entstanden ist, die Ausgangsbedingungen im Nachhinein kaum mehr exakt rekonstruiert werden können. Zudem zeigt sich, dass nicht nur die Bedingungen im Bereich der Abtragsfläche selbst, sondern auch die der oberhalb angrenzenden Hangbereiche berücksichtigt werden müssen (EIDT \& LÖHMANNSRÖBEN 1996, Löhmannsröвen \& Schauer 1996, Mössmer 1985, S. 76), was eine zusätzliche Herausforderung auch für die geostatistische Bearbeitung der Daten bedeutet.

Wenn man die aktuelle Zunahme der Denudation besser verstehen will, lohnt ein Blick in die wechselhafte Geschichte dieser Prozesse im Alpenraum, die eng mit dem Menschen verbunden ist. Es ist davon auszugehen, dass großflächige Rodungen im Mittelalter - im Karwendel zum Beispiel setzten die Rodungen bereits im 6. Jh. ein (Blechschmidt 1990, S. 32) - die Stabilität der Hänge drastisch verminderten. Mit den Rodungen für den Holzbedarf oder die Gewinnung von Weide- und Wiesengrund gingen eine effizient schützende Vegetationsdecke und eine oft tief reichende Durchwurzelung verloren (Blechschmidt 1990, Riedl 1982, Stahr 1997). Die Rodungen führten ebenso zu einer Veränderung des Hangwasserhaushalts, auch wenn diese weniger offensichtlich zutage trat (GEITNER 1999, S. 186f.). Die anthropogen bedingten Veränderungen der Hangsysteme (VEIT 2002, S. 189) machten diese anfälliger gegenüber Denudations- und Erosionsprozessen. Die bereits in Kapitel 3.5 beschriebenen Intensitätsverschiebungen der Landnutzung der jüngsten Vergangenheit ziehen eine Veränderung der Vegetationszusammensetzung nach sich, welche sich auch auf die Beschaffenheit der Böden und deren Stabilität und damit auf das Abtragsgeschehen der Hänge auswirkt. 


\section{Synthese, offene Fragen und Forschungsbedarf}

Die räumliche Verteilung der Studien zur flachgründigen Denudation belegt, dass diese Abtragungsform weit verbreitet auftrifft (vgl. Abb. 1). Aus der Mehrheit der Arbeiten geht eine Zunahme dieser Abtragserscheinung auf Grünlandflächen innerhalb der letzten Jahrzehnte hervor (vgl. Tab. 3). Es besteht auch weitgehend Übereinstimmung unter den Autoren, dass ein Zusammenhang zwischen den weit verbreiteten Extensivierungsmaßnahmen und dem gehäuften Auftreten flachgründiger Denudation besteht. Denn die Nutzung ist die einzige Einflussgröße, die in den letzten Dekaden fast im gesamten Alpenraum erheblichen Veränderungen unterworfen war (BERNHAUPT 1980, TASSER et al. 2005b), wohingegen die übrigen Parameter wie Gestein, geomorphologische Rahmenbedingungen und Böden innerhalb eines solchen Zeitraumes als weitgehend stabil zu bewerten sind. Entgegen dieser Argumentation konnte STOCKER (1985) in den Zentralalpen keinen Zusammenhang zwischen dem Auftreten flacher Abtragserscheinungen und dem strukturellen Wandel nachweisen.

Abweichende Auffassungen bestehen vor allem im Hinblick auf das Prozessverständnis und die Gewichtung der beteiligten Parameter sowie die zu ergreifenden Maßnahmen, wobei es methodisch sinnvoll erscheint, zwischen Ereignissen durch Extremniederschläge und solchen, die auch bei eher durchschnittlichen Witterungsbedingungen vorkommen können, zu unterscheiden. Beim aktuellen Kenntnisstand ist kaum abzuschätzen, ob und wie der Klimawandel die flachgründige Denudation beeinflusst. Entscheidender als die Temperaturen sind sicher die Niederschlagsverhältnisse, seien es die Stark- oder Dauerniederschläge, oder die Bedingungen für Aufbau und Andauer der Schneedecke. Diese sind jedoch generell schwer zu prognostizieren und variieren regional stark. Auch wenn in Fallstudien belegt sein sollte, dass beispielsweise die Starkniederschläge signifikant zugenommen haben - wie es Meusburger \& Alewell (2008) für ihr Testgebiet Urserental zeigen konnten -, ist es methodisch oftmals kaum möglich, diesen Einfluss von dem der Nutzungsextensivierung zu trennen. Denn auch im Urserental war die Zunahme der Abtragsflächen weitgehend auf Flächen extensivierter Nutzung begrenzt (Meusburger \& Alewell 2008).

Aufgrund des nicht ausreichenden Prozessverständnisses sind die Prognosen künftiger Denudationsereignisse und damit auch die Ausweisung und der Schutz von gefährdeten Gebieten, wie sie das Protokoll „Bodenschutz“ der Alpenkonvention fordert (CIPRA 1998), noch mit zu großen Unsicherheiten belegt. Um diese weiter reduzieren zu können, sind zusätzliche prozessorientierte Fallstudien im Detailmaßstab nötig, vor allem mit substrat- oder bodenphysikalischen Messungen und kontinuierlicher Erfassung des Bodenwasserhaushalts. Auch die Auswertung hoch aufgelöster Reliefdaten, wie sie auch für die Alpen mehr und mehr vorliegen (z.B. KRINGER et al. 2009), kann - insbesondere, wenn sie mit differenzierten Substratinformationen kombiniert werden - zum Verständnis der Prozesse beitragen .

Um die kritische Faktorenkombination und ihre räumliche Variabilität besser beschreiben zu können, sollte eine systematische Zusammenstellung von Einzelereignissen aus weiten Teilen des Alpenraums angestrebt werden. Für die statistische Auswertung wären die Eindeutigkeit und Einheitlichkeit dieser Daten essenziell. Es 
bedarf dafür sowohl der Sichtung und Harmonisierung vorhandener Informationen als auch der Aufnahme weiterer Ereignisse. Dabei ist nicht nur auf die nötige Differenzierung nach schurf- und rutschungsbedingten Abtragsformen zu achten, sondern ebenso auf einheitliche Standards für die Erfassung ergänzender Parameter (u.a. Lage, Reliefeinbindung, Gestein, Boden, Vegetation). Wichtig ist ebenso, die Hangbereiche ober- und unterhalb der Denudationsfläche zu charakterisieren und mögliche Angriffsstellen der Abtragung in näherer Umgebung (Zugrisse, Trittschäden) zu erfassen, wie es bei verschiedenen Ereignisdokumentationen schon gemacht wird (z.B. ANDRECS et al. 2002, MARKART et al. 2007, RicKLI \& BUCHER 2003).

Niederschlagsdaten sowie Informationen zur Landnutzungsänderung sind für alle weiteren Auswertungen von besonderer Bedeutung. Ein Vergleich lokaler Einzelereignisse sollte mit einer entsprechend räumlich differenzierten Analyse der Niederschlagsdaten einhergehen. Das ist zumindest bei einzelnen Ereignissen möglich, die zu größeren Schäden führten und daher für bestimmte Regionen gut dokumentiert sind (z.B. Andrecs et al. 2002, Rickli \& Bucher 2003). Bei Auswertungen dieser Extremereignisse sollten - zumindest selektiv - auch Flächen mit einbezogen werden, die scheinbar eine hohe Disposition zur Abtragung aufweisen, dennoch nicht mit Denudation reagiert haben.

Eine große Unsicherheit werden weiterhin die hydrologischen Verhältnisse des oberflächennahen Untergrundes, die Hangwasserdynamik, darstellen. Auch wenn es dazu Detailstudien mit groß angelegten Feldexperimenten (z.B. Projekt CCES-TRAMM der Eidgenössischen Forschungsanstalt für Wald, Schnee und Landschaft) - auch in Bezug auf die Auslösung von Rutschungen - gibt, können diese nur schwer auf andere Lokalitäten übertragen werden. Da es nicht möglich ist, diese Daten in größerem Stil aufzunehmen, sollte verstärkt versucht werden, über die Optimierung von Substratund Reliefdaten boden- und substrathydrologische Informationen abzuleiten. Hierzu sind weiterhin umfangreiche Kartierungen im Gelände unerlässlich, wenngleich neue Verfahren aus der Geophysik und Fernerkundung wichtige Zusatzdaten zu bieten vermögen. Durch neue Datierungsmethoden ist zudem die Abschätzung der längerfristigen Dimensionen von Umlagerungsprozessen möglich geworden (Konz et al. 2009), was wichtig ist, um die aktuellen Prozesse in eine landschaftsgeschichtliche Relation zu setzen.

Wenige Informationen liegen im Hinblick auf die Weiterformung der Denudationsflächen vor, obgleich die hierbei erreichten Abtragssummen nicht unerheblich sein dürften. Die Frage, unter welchen Bedingungen sich die betroffenen Flächen vergrößern oder sich durch eine Wiederbesiedlung von Pflanzen stabilisieren, ist auch im Hinblick auf die anzuwendenden Maßnahmen von großem Interesse.

Die hier nur angedeuteten Forschungsaufgaben umfassen verschiedene Maßstabsbereiche und ein dementsprechend weites Methodenspektrum. Zudem sollten die Studien interdisziplinär ausgerichtet sein, um dem komplexen Zusammenspiel der Parameter in Raum und Zeit gerecht werden zu können. Im Hinblick auf die Zukunft ist anzustreben, den Forderungen des Protokolls zur Durchführung der Alpenkonvention von 1991 im Bereich Bodenschutz (CIPRA 1998) nachzukommen, indem ein länderübergreifendes Bodenerosionsmonitoring für die Alpen entwickelt und aufgebaut wird. 
Dies sollte in enger Abstimmung mit allen beteiligen Partnern aus Wissenschaft und Praxis erfolgen, damit die Ergebnisse sinnvoll vergleichbar sind. Mit dieser Maßnahme wäre nicht nur eine verlässliche Kontrolle über mögliche Veränderungen gegeben, die erhobenen Daten würden von Jahr zu Jahr eine zunehmend solidere Grundlage für wissenschaftliche Auswertungen hinsichtlich der Prozesszusammenhänge bieten. Nur so können langfristig verlässliche Aussagen über den Einfluss des globalen Wandels auf die Landschaft und die Ökosysteme des Alpenraums getroffen werden.

\section{Danksagung}

Der philosophisch-historischen Klasse der Österreichischen Akademie der Wissenschaften sei für die Unterstützung der Dissertation durch die Bereitstellung eines Stipendiums gedankt. Außerdem bedanken sich die Autoren für die konstruktiven Hinweise der beiden anonymen Gutachter.

\section{Literaturverzeichnis}

Abellan A. et al. (2009), Detection of millimetric deformation using a terrestrial laser scanner experiment and application to a rockfall event. In: Natural Hazards and Earth System Sciences, 9, S. 365-372.

Ahnert F. (1999), Einführung in die Geomorphologie. Stuttgart, Eugen Ulmer.

Alewell C. et al. (2008), Methods to describe and predict soil erosion in mountain regions. In: Landscape and Urban Planning, 88, S. 46-53.

AndRECs P. et al. (2002), Untersuchung der Rutschprozesse vom Mai 1999 im Laternsertal (Vorarlberg). In: Schriftenreihe d. Bundesamtes u. Forschungszentrums f. Wald, 127, S. 55-87.

Auerswald K. (1998), Die Bodenerosion durch Wasser. In: Richter G. (Hrsg.), Bodenerosion und Bodenschutz - Analyse und Bilanz eines Umweltproblems. S. 33-42. Darmstadt, Wiss. Buchges.

Avanzi G., Giannecchini R., Puccinelli A. (2004), The influence of the geological and geomorphological settings on shallow landslides. An example in a temperate climate environment: the June 19, 1996 event in northwestern Tuscany (Italy). In: Engineering Geology, 73, S. 215-228.

B athurst J., Bovolo I., Cisneros F. (2010), Modelling the effect of forest cover on shallow landslides at the river basin scale. In: Ecological Engineering, 36, S. 317-327.

B ̈̈TZING W. (2003), Die Alpen - Geschichte und Zukunft einer europäischen Kulturlandschaft. München, C.H. Beck.

Becker A. et al. (2007), Ecological and Land Use Studies Along Elevational Gradients. In: Mountain Research and Development, 27, 1, S. 58-65.

Begueria S. (2006), Changes in land cover and shallow landslide activity: A case study in the Spanish Pyrenees. In: Geomorphology, 74, S. 196-206.

Bernhaupt P. (1980), Zum Problem der Bodenerosion in Almgebieten am Beispiel der Planneralm, Wölzer Tauern, Steiermark. In: Interpraevent 1980 Tagungspubl., 1, S. 291-308.

Birkenhauer J. (2001), Blaiken in den Alpen - Ursachen und Verbreitung. In: Mitt. d. Geogr. Ges. in München, 85, S. 1-17. 
Bitelli G., Dubbini M., Zanutta A. (2004), Terrestrial laser scanning and digital photogrammetry to monitor landslide bodies. In: Proc. of the $\mathrm{XX}^{\text {th }}$ ISPRS Congress, Istanbul, Vol. XXXV, part B5, S. 246-251.

Blechschmidt G. (1990), Die Blaikenbildung im Karwendel. In: Jahrbuch d. Vereins z. Schutz d. Bergwelt, 55, S. 31-45.

BRooks S.M. et al. (2002), Regolith stripping and the control of shallow translational hillslope failure: application of a two-dimensional coupled soil hydrology-slope stability model, Hawke's Bay, New Zealand. In: Geomorphology, 45, S. 165-179.

Bunza G. et al. (1996), Abfluss und Abtragsprozesse in Wildbacheinzugsgebieten - Grundlagen zum integralen Wildbachschutz (= Schriftenreihe d. Bayer. Landesamtes f. Wasserwirtschaft, 27), S. 90.

CAINE N. (1980), The rainfall intensity - duration control of shallow landslides and debris flows. In: Geografiska Annaler, 62 A, 1-2, S. 23-27.

Ceriani M., Lauzi S., Padovan N. (1992), Rainfalls and landslides in the alpine area of Lombardia Region - Central Alps - Italy. In: Interpraevent 1992 Tagungspubl., 2, S. 9-20.

CIPRA - Internationale Alpenkonvention (1998), Protokoll zur Durchführung der Alpenkonvention von $1991 \mathrm{im}$ Bereich Bodenschutz.

DeRose R.C., Thomson N.A., Roberts A.H.C. (1995), Effect of landslide erosion on Taranaki hill pasture production and composition. In: New Zealand Journal of Agricultural Research, 38, S. 457-471.

Dikau R. (2004), Mass Movement. In: Goudie A. (Hrsg.), Encyclopedia of Geomorphology, S. 644-652. London, Routledge.

Douglas G.M., Trustrum N.A., Brown I.C. (1986), Effect of soil slip erosion on Wairoa hill pasture production and composition. In: New Zealand Journal of Agriculture Research, 29, S. 183-192.

Dymond J.R., Jessen M.R., Lovell L.R. (1999), Computer simulation of shallow landsliding in New Zealand hill country. In: Intern. Journal of Applied Earth Observation and Geoinformation, 1, 1, S. 122-131.

EIDT M., LÖHMANNSRÖBEN R. (1996), Zusammenhänge zwischen bodenkundlichen Standortfaktoren und verschiedenen Abtragsformen. In: Interpraevent 1996 Tagungspubl., 1, S. 247-261.

Ellenberg H. (1996), Vegetation Mitteleuropas mit den Alpen in ökologischer, dynamischer und historischer Sicht. Stuttgart, Eugen Ulmer.

EwALD J. (1996), Graslahner - Rasengesellschaften in der montanen Waldstufe der Tegernseer Kalkalpen. In: Berichte d. Bayer. Botan. Ges., 66/67, S. 115-133.

GaLl H. (1947, publiziert und mit einer Nachlese versehen 1985), Bodenerosion und Bodenerhaltung im Gebirgsland (Am besonderen Beispiel Nordtirols). Diss., Geogr. Inst., Univ. Innsbruck.

GAMPER S., TASSER E. (2002), Soil development depending on land use and vegetation changes in sub-alpine areas. In: BotTARin R., TAPPEINER U. (Hrsg.), Interdisciplinary Mountain Research, S. 180-191. Berlin, Blackwell Science.

Garcia-Ruiz J.M. et al. (1996), Land-use changes and sustainable development in mountain areas: a case study in the Spanish Pyrenees. In: Landscape Ecology, 11, S. 267-277.

GeITNER C. (1999), Sedimentologische und vegetationsgeschichtliche Untersuchungen an fluvialen Sedimenten in den Hochlagen des Horlachtales (Stubaier Alpen/Tirol) - Ein Beitrag zur zeitlichen Differenzierung der fluvialen Dynamik im Holozän (= Münchener Geogr. Abhandlungen, 32).

Geitner C., Tusch M. (2008), Bodenschutz in den Alpen: Das Projekt SEPP (Soil Evaluation in Planning Procedures) und das Protokoll „Bodenschutz“ der Alpenkonvention. In: Jahresbericht d. Innsbrucker Geogr. Ges., S. 129-141. 
Giannecchini R. (2005), Rainfall triggering soil slips in the southern Apuan Alps (Tuscany, Italy). In: Advances in Geosciences, 2, S. 21-24.

Glade T. (1998), Establishing the frequency and magnitude of landslide-triggering rainstorm events in New Zealand. In: Environmental Geology, 35, 2-3, S. 160-174.

Hamberger M., Moser M. (2008), Der Einsatz verschiedener Klassifikatoren zur Erkennung flachschaliger Rutschungen - Modellgebiet Sachseln/Schweiz. In: Interpraevent 2008 Conf. Proc., 2, S. 159-170.

Hiura H. (1988), Hazard mapping in use of surface slide transition model. In: Interpraevent 1988 Tagungspubl., 1, S. 297-313.

Ibsen M.-L., Brunsden D. (1996), Slab Slide. In: Dikau R. et al. (Hrsg.), Landslide Recognition - Identification, Movement and Causes, S. 78-84. Chichester, Wiley.

IN DER GAND H. (1972), Wald als Lawinenschutz. Weissfluhjoch/Davos, Eidgen. Inst. f. Schneeu. Lawinenforschung.

KARL J. (1961), Blaikenbildung auf Allgäuer Blumenbergen. In: Jahrbuch d. Vereins z. Schutze d. Alpenpflanzen u. -tiere, 26, S. 55-62.

Kelch G., Drexler O., Zech W. (1977), Über den Bodenabtrag im Kampenwandgebiet. Versuch einer quantitativen Erfassung der Zusammenhänge zwischen Bodenabtrag und den Faktoren Höhenlage, Exposition, Hangneigung, Muttergestein und Bodennutzung. In: Zeitschrift f. Geomorphologie, Suppl. 28, S. 134-147.

Körner C. (1999), Alpine Plant Life: Functional Plant Ecology of High Mountain Ecosystems. Berlin - Heidelberg, Springer.

Konz N. et al. (2009), Cesium-137-based erosion-rate determination of a steep mountainous region. In: Journal of Plant Nutrition and Soil Science, 172, S. 615-622.

Kringer K. et al. (2009), Geomorphometric Analyses of LiDAR Digital Terrain Models for Digital Soil Mapping. In: Proc. of Geomorphometry, Zürich, 31.8.-2.9. 2009, S. 74-81.

LaAtsch W., Baum U. (1976), Faktoren der Wald- und Bodenzerstörung durch Schnee in den Alpen. In: Agrochimica, 20, S. 324-338.

Laatsch W., Grottenthaler W. (1972), Typen von Massenverlagerungen in den Alpen und ihre Klassifizierung. In: Forstwiss. Centralblatt, 91, S. 309-339.

Laatsch W., Grottenthaler W. (1973), Labilität und Sanierung der Hänge in der Alpenregion des Landkreises Miesbach. München, Bayer. Staatsministerium f. Ernährung, Landwirtschaft u. Forsten.

Lateltin O. et al. (2005), Landslide risk management in Switzerland. In: Landslides, 2, S. 313-320.

Leitinger G. et al. (2008), Development and validation of a spatial snow-gliding model. In: Ecological Modelling, 211, S. 363-374.

Lichtenegger E. (1988), Einfluss der Almdüngung auf den Pflanzenbestand. In: Alm- u. Bergbauer, 38, 5, S. 204-218

LÖHMANNSRÖBEN R. (2002), Die Bedeutung des Bodens im Zusammenhang mit der hydrologischen Regionalisierung. In: Wiener Mitt.: Niederschlag-Abfluss Modellierung - Simulation und Prognose, 164, S. 201-213.

LÖHMANNSRÖBEN R., SCHAUER T. (1996), Ableitung hydrologischer Eigenschaften zur Beurteilung des Abfluss- und Abtragsgeschehens aus boden- und vegetationskundlichen Kriterien. In: Interpraevent 1996 Tagungspubl., 1, S. 99-112.

MaAg S., Nösberger J., Lüschner A. (2001), Mögliche Folgen einer Bewirtschaftungsaufgabe von Wiesen und Weiden im Berggebiet. Ergebnisse des Komponentenprojektes D, Polyprojekt PRIMALP ETH Zürich.

MACDonald D. et al. (2000), Agricultural abandonment in mountain areas of Europe: Environmental consequences and policy response. In: Journal of Environmental Management, 59, S. 47-69. 
MARKART G. et al. (2006), Geländeanleitung zur Abschätzung des Oberflächenabflussbeiwertes bei Starkregen - Grundzüge und erste Erfahrungen. In: Wiener Mitt.: Wasser-AbwasserGewässer, 197, S. 159-178.

Markart G. et al. (2007), 22. und 23. August 2005 - Analyse von Hochwasser- und Rutschungsereignissen in ausgewählten Gemeinden Vorarlbergs (= Schriftenreihe d. Bundesforschungs- u. Ausbildungszentrums f. Wald, Naturgefahren u. Landschaft, 5).

Marui H. (1984), Die Mechanik oberflächennaher Bewegungen. In: Interpraevent 1984 Tagungspubl., 3, S. 141-156.

Meusburger K., Alewell C. (2008), Impacts of anthropogenic and environmental factors on the occurrence of shallow landslides in an alpine catchment (Urseren Valley, Switzerland). In: Natural Hazards and Earth System Sciences, 8, S. 509-520.

Mössmer E. (1984), Unterschiede der standörtlichen Einflussfaktoren auf die Blaikenerosion zwischen beweideten und aufgelassenen Almen. In: Interpraevent 1984 Tagungspubl., 3, S. 79-88.

Mössmer E. (1985), Einflussfaktoren für die Blaikenerosion auf beweideten und aufgelassenen Almflächen im kalkalpinen Bereich der Landkreise Miesbach und Rosenheim. In: Forstl. Forschungsberichte München, 63, S. 1-137.

Morgan R.P.C. (2005), Soil erosion \& Conservation. London, Blackwell Science.

Newesely C. et al. (2000), Effects of land use changes on snow gliding processes in alpine ecosystems. In: Basic and Applied Ecology, 1, S. 61-67.

PARry M.L. et al. (Hrsg.) (2007), Climate Change 2007: Impacts, Adaptations and Vulnerability. Contribution of Working Group II to the Fourth Assessment Report of the Intergovernmental Panel on Climate Change. Cambridge, Cambridge Univ. Press.

Psenner R. (2008), Wasser aus den Alpen Globaler Wandel - regionale Anpassung. In: LacKNer R., Psenner R., Walcher M. (Hrsg.), Ist es der Sindtfluss? Kulturelle Strategien \& Reflexionen zur Prävention und Bewältigung von Naturgefahren, S. 25-30. Innsbruck, Innsbruck Univ. Press.

Reisigl H., Keller R. (1994), Alpenpflanzen im Lebensraum - Alpine Rasen- und Felsvegetation. Stuttgart - Jena - New York, Gustav Fischer.

Rickli C. et al. (2002), Wirkung des Waldes auf oberflächennahe Rutschprozesse. In: Schweizer. Zeitschrift f. Forstwesen, 153, 11, S. 437-445.

Rickli C., BuCHER H. (2003), Oberflächennahe Rutschungen, ausgelöst durch die Unwetter vom 15.-16.7.2002 im Napfgebiet und vom 31.8.-1.9.2002 im Gebiet Appenzell. Projektbericht zuhanden d. Bundesamtes f. Wasser u. Geologie BWG.

RiCKLI C. et al. (2004), Untersuchungen zu oberflächennahen Rutschungen des Jahres 2002 im Napfgebiet und in der Region Appenzell. In: Bulletin f. Angewandte Geologie, 9, 1, S. 37-49.

Rickli C., GRAF F. (2009), Effects of forests on shallow landslides - case studies in Switzerland. In: Forest Snow and Landscape Research, 82, 1, S. 33-44.

RIEDL H. (1982), Die Prägekraft des sozioökonomischen Strukturwandels auf Morpho- und Pedosphäre des subalpinen Lebensraumes. In: Mitt. d. Österr. Bodenkundl. Ges., 25, S. 5-51.

SAla O. et al. (2000), Global Biodiversity Scenarios for the Year 2100. In: Science, 287, S. $1770-1774$

SEEber J. et al. (2005), Abundance and trophic structure of macrofauna decomposers on alpine pastureland (Central Alps, Tyrol): effects of abandonment of pasturing. In: Pedobiologia, 49, S. 221-228.

Schauer T. (1975), Die Blaikenbildung in den Alpen (= Schriftenreihe d. Bayer. Landesamtes f. Wasserwirtschaft, 1). München. 
Schauer T. (1999), Beispiele von Erosionsprozessen in Zusammenhang mit den Standortfaktoren Nutzung und Vegetation im bayerischen Alpenraum. In: Fischer K. (Hrsg.), Massenbewegungen und Massentransporte in den Alpen als Gefahrenpotential (= Boden, Relief, Paläoklima, 14), S. 117-128.

StAHR A. (1997), Bodenkundliche Aspekte der Blaikenbildung auf Almen (= Nationalpark Berchtesgaden, Forschungsberichte, 39).

STOCKER E. (1985), Zur Morphodynamik von „Plaiken“ - Erscheinungsformen beschleunigter Hangabtragung in den Ostalpen, anhand von Messungsergebnissen aus der Kreuzeckgruppe, Kärnten. In: Mitt. d. Österr. Geogr. Ges., 127, S. 44-70.

StOcKer E. (2005), Blaiken - Räumlich-zeitliche Veränderung an Systemen beschleunigter Abtragung im Umkreis der Waldgrenze am Beispiel der Kreuzeckgruppe, Kärnten (= Salzburger Geogr. Arbeiten, 38), S. 53-68.

TAPPEINER U., TASSER E., TAPPEINER G. (1998), Modelling vegetation patterns using natural and anthropogenic influence factors: preliminary experience with a GIS based model applied to an Alpine area. In: Ecological Modelling, 113, S. 225-237.

TAPPEINER U. et al. (2008), Effects of historical and likely future scenarios of land use on above- and belowground vegetation carbon stocks of an alpine valley. In: Ecosystems, 11, S. $1383-1400$

TAsser E. et al. (1999), Potential risks through land-use changes. In: Cernusca A., TAPPeiner U., BAYField N. (Hrsg.), Land-Use Changes in European Mountain Ecosystems. ECOMONT - Concept and Results, S. 235-246.

TASSER E., TAPPEINER U. (2002), Impact of land use changes on mountain vegetation. In: Applied Vegetation Science, 5, S. 173-184.

Tasser E., Mader M., Tappeiner U. (2003), Effects of land use in alpine grasslands on the probability of landslides. In: Basic and Applied Ecology, 4, S. 271-280.

Tasser E., Tappeiner U., Cernusca A. (2005a), Ecological Effects of Land-use Changes in the European Alps. In: Huber U., Bugmann H., Reasoner M. (Hrsg.), Global Change and Mountain Regions, S. 409-420. Dordrecht, Springer.

Tasser E., Mader M., TAppeiner U. (2005b), Auswirkungen von Bewirtschaftungsänderungen auf die Blaikenbildung im Gebirge. In: Mitt. d. Österr. Bodenkundl. Ges., 72, S. 193-217.

UNCED (Konferenz der Vereinten Nationen für Umwelt und Entwicklung) (1992), Agenda 21. Rio de Janeiro.

VeIT H. (2002), Die Alpen - Geoökologie und Landschaftsentwicklung. Stuttgart, Eugen Ulmer.

Veit H., Mailänder R., Vonlanthen C. (2002), Periglaziale Deckschichten im Alpenraum: bodenkundliche und landschaftsgeschichtliche Bedeutung. In: PGM, 146, S. 6-14.

Witzig J., Badoux A., Hegg C., Lüscher P. (2004), Waldwirkung und Hochwasserschutz - eine standörtlich differenzierte Betrachtung. In: Forst u. Holz, 59, 10, S. 476-479.

Zepr H. (2004), Geomorphologie - Eine Einführung. Paderborn, Ferdinand Schöningh.

ZweckL J., SPANDAu L. (1987), Untersuchungen zur Blaikenerosion im Jennergebiet des Alpenparks Berchtesgaden. In: Landschaft u. Stadt, 19, S. 122-128. 